\title{
Use of N-N Bond Stereodynamics in Ring Closing Metathesis to Form Medium-Sized Rings and Macrocycles
}

\author{
Yi Jin Kim and Daesung Lee* \\ Department of Chemistry, University of Wisconsin-Madison, Madison, WI 53706
}

\section{Supporting Information}

\section{Materials and Methods}

Compounds were purchased from Aldrich. DMF was distilled from $\mathrm{MgSO}_{4}$. THF was distilled from sodium/benzophenone and methylene chloride was distilled from $\mathrm{CaH}_{2}$. Flash chromatography was performed using silica gel $60 \AA$ (32-63 mesh) from Sorbent Technologies. Reactions were monitored by thin layer chromatography (TLC) using $0.25 \mathrm{~mm}$ E. Merk precoated silica gel $60 \mathrm{~F}_{254}$ (particle size $0.040-0.063 \mathrm{~mm}$ ). ${ }^{1} \mathrm{H}$ NMR and ${ }^{13} \mathrm{C}$ NMR spectra were recorded on a Bruker AC-300 or a Varian INOVA-500 spectrometer. Chemical shifts are reported in $\delta$ values relative to the internal standard tetramethyl silane (TMS) or to residual solvent peaks. 


\section{Experimental}

Representative procedure for the synthesis of $\mathbf{2 a}-\mathbf{2} \mathbf{i}$

Synthesis of 2a

To a mixture of 2,2-Dimethyl-4-pentenal $(0.55 \mathrm{ml}, 3.7 \mathrm{mmol})$ and dibenzylazodicarboxylate $(1.1$ g, $3.7 \mathrm{mmol}$ ) was added rhodium (II) acetate dimer (33 $\mathrm{mg}, 0.07 \mathrm{mmol}, 2 \mathrm{~mol} \%$ ), and the reaction mixture was allowed to stir for 24 hours at room temperature. The mixture was purified by silica gel chromatography (4:1 hexane:ethyl acetate), yielding a yellowish oil (754 mg, $49 \%$ ). This alkenyl hydrazine derivative 6 (195 mg, $0.48 \mathrm{mmol})$ was dissolved in DMF $(2.0 \mathrm{~mL})$ and to this solution was then added $\mathrm{Cs}_{2} \mathrm{CO}_{3}(185 \mathrm{mg}, 0.57 \mathrm{mmol})$, followed by excess allyl bromide $(0.08 \mathrm{~mL}, 0.96 \mathrm{mmol})$. The reaction was stirred at room temperature for approximately 2 hours. The reaction mixture was diluted with ethyl acetate $(2.0 \mathrm{~mL})$ and washed with water $(5 \times 2.0$ $\mathrm{mL}$ ) to remove residual DMF. The combined organic layer was dried over $\mathrm{MgSO}_{4}$ and concentrated in vacuo. The residue was then purified by silica gel chromatography (5:1 hexane:ethyl acetate), yielding 2a as a colorless oil (112 mg, $52 \%)$.

Representative procedure for the formation of cyclic hydrazine derivatives $\mathbf{3 a}-\mathbf{3 g}$ and $\mathbf{1 1}$ via RCM

Diene 2a (30 mg, $0.07 \mathrm{mmol})$ was dissolved in $\mathrm{CH}_{2} \mathrm{Cl}_{2}(0.002 \mathrm{M})$ in a $100 \mathrm{ml}$ round bottom flask and the solution was flushed with $\mathrm{N}_{2}$ for approximately 5 minutes. The flask was fitted with a reflux condenser and Grubbs second generation catalyst ( $5 \mathrm{~mol} \%$ dissolved in $1 \mathrm{~mL}$ of $\mathrm{CH}_{2} \mathrm{Cl}_{2}$ ) was added. The reaction was refluxed for 2 hours under $\mathrm{N}_{2}$. The solution was then cooled to room temperature in open atmosphere and the solvent was removed in vacuo. The crude material was purified by silica gel chromatography (4:1 hexane:ethyl acetate) to yield 3a as a yellowish oil (26 mg, $93 \%)$.

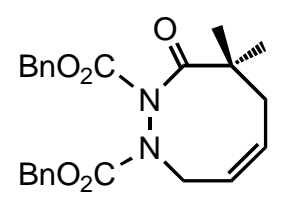

$3 a$ 
3a: ${ }^{1}$ H NMR (300 MHz, CDCl $)_{3}$ major rotamer: $\delta 7.31(\mathrm{~m}, 10 \mathrm{H}), 5.95(\mathrm{~m}, 1 \mathrm{H}), 5.49(\mathrm{~m}, 1 \mathrm{H})$, $5.20,5.15,5.12,5.04(\mathrm{~d}, J=12.2,4 \mathrm{H}), 4.88(\mathrm{dt}, J=16.6,2.7,1 \mathrm{H}), 3.95,3.87$ (2 sets of rotamers, $\mathrm{dd}, J=16.6,6.0,1 \mathrm{H}), 2.81$ (overlapping rotamers, $\mathrm{dd}, J=13.3,9.4,1 \mathrm{H}$ ), 1.89 (dd, $J=13.3,7.8$, $1 \mathrm{H}), 1.31(\mathrm{~s}, 3 \mathrm{H}), 1.14(\mathrm{~s}, 3 \mathrm{H})$; minor rotamer: $\delta 5.43(\mathrm{~m}, 1 \mathrm{H}), 5.32,5.29,5.27,5.22(\mathrm{~d}, J=$ $13.3,4 \mathrm{H}), 4.79(\mathrm{dt}, J=16.6,2.7,1 \mathrm{H}), 1.31(\mathrm{~s}, 3 \mathrm{H}), 1.23(\mathrm{~s}, 3 \mathrm{H})$.

${ }^{13}$ C NMR (75 MHz, $\mathbf{C D C l}_{3}$ ) major rotamer: $\delta$ 176.89, 155.36, 151.85, 134.85, 134.61, 130.09, 128.40, 128.34, 128.06, 127.94, 127.69, 68.85, 68.38, 47.83, 47.45, 38.30, 28.33, 26.85; minor rotamer: $\delta 177.04,152.19,135.29,134.81,128.43,128.28,128.24,128.11,128.00,69.00$, 68.69, 48.77, 47.66, 38.45, 28.24, 26.72; HRMS calcd for $\mathrm{C}_{24} \mathrm{H}_{26} \mathrm{~N}_{2} \mathrm{O}_{5}\left[\mathrm{MNa}^{+}\right]: 445.1739$ Found: 445.1737.

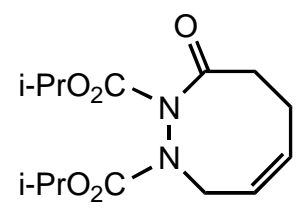

$3 b$

3b: ${ }^{1} \mathbf{H}$ NMR (300 MHz, $\mathbf{C D C l}_{3}$ ) major rotamer: $\delta 5.94(\mathrm{~m}, 1 \mathrm{H}), 5.58(\mathrm{~m}, 1 \mathrm{H}), 5.00-5.18$ (overlapping septets, $J=6.2,2 \mathrm{H}), 4.91(\mathrm{~d}, J=16.7,1 \mathrm{H}), 3.83(\mathrm{dd}, J=16.9,5.3,1 \mathrm{H}), 2.94(\mathrm{~m}$, $1 \mathrm{H}), 2.66(\mathrm{~m}, 2 \mathrm{H}), 2.30(\mathrm{~m}, 1 \mathrm{H}), 1.37(\mathrm{~d}, J=6.2,3 \mathrm{H}), 1.35(\mathrm{~d}, J=6.2,3 \mathrm{H}), 1.31(\mathrm{~d}, J=6.2,3 \mathrm{H})$, $1.27(\mathrm{~d}, J=6.2,3 \mathrm{H})$; minor rotamer: $\delta 5.65-5.78(\mathrm{~m}, 2 \mathrm{H}), 4.95-5.01(\mathrm{~m}, 1 \mathrm{H}), 4.77(\mathrm{~d}, J=$ $16.7,1 \mathrm{H}), 3.90(\mathrm{dd}, J=16.9,5.3,1 \mathrm{H}), 2.16(\mathrm{~m}, 1 \mathrm{H}), 1.17-1.40$ (overlapping d, $J=6.2,9 \mathrm{H}$ ).

${ }^{13}$ C NMR (75 MHz, CDCl 3 ) major rotamer: $\delta$ 173.68, 154.85, 150.40, 130.91, 124.84, 72.14, $71.17,47.23,37.23,23.54,22.29,22.11,21.72,21.70$; minor rotamer: $\delta 131.16,124.96,71.22$, 48.26, 37.31, 23.44, 22.16, 22.08, 22.09; HRMS calcd for $\mathrm{C}_{14} \mathrm{H}_{22} \mathrm{~N}_{2} \mathrm{O}_{5}\left[\mathrm{MNa}^{+}\right]$: 321.1426 Found: 321.1432 .

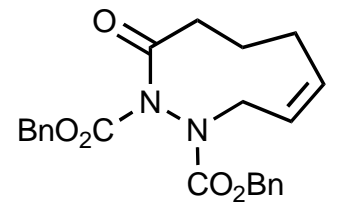

3c

3c: ${ }^{1}$ H NMR (500 MHz, $\left.\mathbf{C D C l}_{3}\right)$ major rotamer: $\delta 7.20-7.40(\mathrm{~m}, 10 \mathrm{H}), 5.86(\mathrm{~m}, 1 \mathrm{H}), 5.71(\mathrm{~m}$, 1H), 5.22, 5.11, 5.10, 5.05 (d, $J=12.2,4 \mathrm{H}), 4.46$ (dd, $J=13.4,6.8,1 \mathrm{H}), 3.79$ (dd, $J=13.4,7.6$, 
$1 \mathrm{H}), 2.61(\mathrm{td}, J=12.7,3.5,1 \mathrm{H}), 2.27(\mathrm{ddd}, J=12.7,5.0,3.4,1 \mathrm{H}), 2.17(\mathrm{~m}, 2 \mathrm{H}), 1.98(\mathrm{~m}, 1 \mathrm{H})$, $1.81(\mathrm{~m}, 1 \mathrm{H})$; minor rotamer: $\delta 5.32,5.27,5.26,5.21(\mathrm{~d}, J=12.2,4 \mathrm{H}), 4.41(\mathrm{dd}, J=13.4,6.8$, $1 \mathrm{H}), 3.85(\mathrm{dd}, J=13.4,7.6,1 \mathrm{H}), 2.36(\mathrm{ddd}, J=12.7,5.0,3.4,1 \mathrm{H})$.

${ }^{13}$ C NMR (125 MHz, CDCl $)$ major rotamer: $\delta$ 175.31, 154.84, 151.04, 137.51, 135.40, $134.68,128.58,128.51,128.04,127.93,127.88,127.75,124.01,69.02$, 68.31, 46.72, 33.28, 27.22, 25.63; minor rotamer: $\delta 155.25,151.40,135.48,134.85,128.62,128.56,128.50,128.44$, $128.35,128.31,124.15,69.15,68.70,47.04,33.39,27.29,25.54$; HRMS calcd for $\mathrm{C}_{23} \mathrm{H}_{24} \mathrm{~N}_{2} \mathrm{O}_{5}$ $\left[\mathrm{MNa}^{+}\right]$: 431.1583 Found 431.1594.

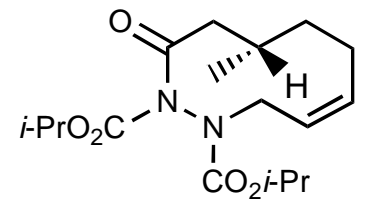

3d

3d: ${ }^{1} \mathbf{H}$ NMR (300 MHz, $\mathbf{C D C l}_{3}$ ) major rotamer: $\delta 5.67(\mathrm{~m}, 1 \mathrm{H}), 5.53(\mathrm{~m}, 1 \mathrm{H}), 5.03$ (septet, $J$ $=6.2,1 \mathrm{H}), 4.95($ septet $, J=6.2,1 \mathrm{H}), 4.28(\mathrm{dd}, J=13.1,6.5,1 \mathrm{H}), 3.97(\mathrm{dd}, J=13.1,11.0,1 \mathrm{H})$, $2.41(\mathrm{~m}, 1 \mathrm{H}), 2.22$ (dd, $J=14.0,11.4,1 \mathrm{H}), 1.83-2.02$ (br m, 2H), 1.44-1.71 (m, 2H), 1.30 (2 overlapping d, $J=6.2,6 \mathrm{H}), 1.20$ (2 overlapping d, $J=6.2,6 \mathrm{H}), 1.00(\mathrm{~d}, J=7.0,3 \mathrm{H})$; minor rotamer: $\delta 4.19(\mathrm{dd}, J=13.1,6.5,1 \mathrm{H}), 4.04(\mathrm{dd}, J=13.1,11.0,1 \mathrm{H}), 2.23(\mathrm{dd}, J=14.0,11.4$, 1H), 1.24-1.37 (overlapping d, $J=6.2$, isopropyl $\underline{\mathrm{CH}}_{3}$ 's).

${ }^{13}$ C NMR (75 MHz, $\mathbf{C D C l}_{3}$ ) major rotamer: $\delta$ 173.17, 155.40, 152.88, 139.25, 121.42, 71.20, $69.79,46.32,40.51,36.57,29.44,24.24,23.65,21.76,21.66,21.63,21.46$; minor rotamer: 139.133, 121.344, 71.35, 70.23, 48.35, 41.28, 21.88, 21.83, 21.79, 21.56; HRMS calcd for $\mathrm{C}_{17} \mathrm{H}_{28} \mathrm{~N}_{2} \mathrm{O}_{5}\left[\mathrm{MNa}^{+}\right]$: 363.1896 Found: 363.1912 .

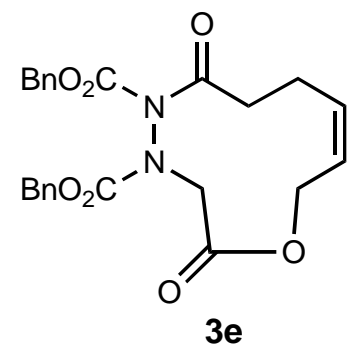


3e: ${ }^{1}$ H NMR (300 MHz, CDCl $)$ major rotamer: $\delta 7.18-7.40(\mathrm{~m}, 10 \mathrm{H}), 5.83(\mathrm{~m}, 2 \mathrm{H}), 5.03$, 5.08, 5.09, $5.18(\mathrm{~d}, J=12.4,4 \mathrm{H}), 4.9(\mathrm{~d}, J=15.5,1 \mathrm{H}), 4.67(\mathrm{~m}, 1 \mathrm{H}), 4.42(\mathrm{~m}, 1 \mathrm{H}), 3.98(\mathrm{~d}, J=$ $15.5,1 \mathrm{H}), 3.65$ (br m, 1H), $2.54(\mathrm{~m}, 1 \mathrm{H}), 2.44$ (m, 2H); minor rotamer: $\delta 5.14,5.23,5.24,5.27$ (d, $J=12.4,4 \mathrm{H}), 4.71(\mathrm{~d}, J=15.5,1 \mathrm{H}), 4.04$ (d, $J=15.5,1 \mathrm{H}), 3.73$ (br m, 1H).

${ }^{13}$ C NMR (75 MHz, $\mathbf{C D C l}_{3}$ ) major rotamer: $\delta$ 172.03, 166.33, 154.84, 152.74, 136.25, 135.59, 134.44, 128.89, 128.86, 128.65, 128.37 127.91, 124.35, 69.60, 68.71, 60.13, 51.87, 36.30, 26.18; minor rotamer: $\delta 171.84,165.99,154.46,153.03,136.63,135.37,134.60,128.98,128.77$, 128.39, 128.28, 128.23, 124.13, 69.71, 69.19, 52.61, 36.27; HRMS calcd for $\mathrm{C}_{24} \mathrm{H}_{24} \mathrm{~N}_{2} \mathrm{O}_{7}$ $\left[\mathrm{MNa}^{+}\right]$: 475.1481 Found: 475.1477.

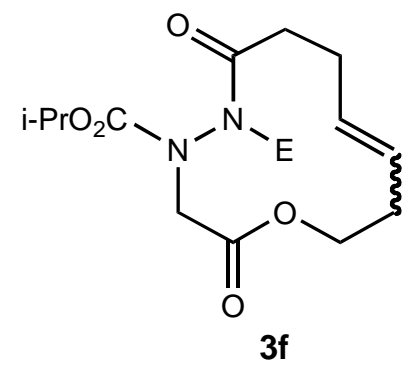

3f: ${ }^{1} \mathbf{H}$ NMR (300 MHz, $\mathbf{C D C l}_{3}$ ) (Z)-isomer (major isomer): $\delta 5.34(\mathrm{~m}, 2 \mathrm{H}), 5.06$ (septet, $J=$ $6.3,1 \mathrm{H}), 4.96$ (septet, $J=6.3,1 \mathrm{H}), 4.65$ (d, $J=16.1$, 1H, major rotamer of (Z)-isomer), 4.52 (d, $J=15.9,1 \mathrm{H}$, minor rotamer of $(\mathrm{Z})$-isomer), $4.27(\mathrm{~m}, 2 \mathrm{H}), 3.18(\mathrm{~m}, 1 \mathrm{H}), 2.68(\mathrm{~m}, 1 \mathrm{H}), 2.48(\mathrm{~m}$, $1 \mathrm{H}), 2.38(\mathrm{~m}, 1 \mathrm{H}), 2.27(\mathrm{~m}, 2 \mathrm{H}), 1.36(\mathrm{~d}, J=6.2,3 \mathrm{H}), 1.30(\mathrm{~d}, J=6.2,3 \mathrm{H}), 1.21(\mathrm{~d}, J=6.2$, $3 \mathrm{H}), 1.20(\mathrm{~d}, J=6.2,3 \mathrm{H})$; (E)-isomer: $\delta 5.61(\mathrm{dd}, J=17.4,8.7,1 \mathrm{H}), 5.34(\mathrm{~m}, 1 \mathrm{H}), 5.05$ (septet, $J=6.3,1 \mathrm{H}), 4.96$ (septet, $J=6.3,1 \mathrm{H}), 4.84$ (d, $J=17.0,1 \mathrm{H}$, major rotamer of (E)-isomer), 4.66 (d, $J=16.6,1 \mathrm{H}$, minor rotamer of (E)-isomer), 4.50 (dd, $J=10.2,2.6,1 \mathrm{H}), 4.43$ (dd, $J=10.2$, 2.6, $1 \mathrm{H}), 4.39(\mathrm{dd}, J=9.7,2.5,1 \mathrm{H}$, minor rotamer), $4.02-4.14$ (m, 3H, mixture of rotamers), $2.85(\mathrm{dd}, J=8.7,3.5,1 \mathrm{H}), 2.80(\mathrm{dd}, J=8.7,3.5,1 \mathrm{H}), 2.67(\mathrm{~m}, 1 \mathrm{H}), 2.32-2.61(\mathrm{~m}, 4 \mathrm{H}$, mixture of rotamers), 1.18-1.39 (overlapping d, $J=6.2,9 \mathrm{H}$ ).

${ }^{13} \mathbf{C}$ NMR (75 MHz, $\mathbf{C D C l}_{3}$ ) (Z)-isomer, major rotamer: $\delta$ 172.04, 167.19, 154.45, 152.37, $130.28,129.44,71.92,70.81,62.54,50.92,36.81,33.15,28.63,21.76,21.76,21.57,21.52$; (E)isomer, major rotamer: $\delta 172.67,167.59,153.92,152.00,130.58,129.16,72.13,71.23,63.56$, 51.27, 36.08, 26.80, 21.85, 21.63, 21.45; mixture of minor rotamers: $\delta 172.61,167.38,167.08$, $152.30,127.39,127.08,72.10,71.45,71.02,63.72,63.56,51.91,51.63,51.27,36.86,36.31$, 
36.08, 29.49, 28.57, 26.80, 26.71, 23.04, 23.18, 21.85, 21.63, 21.45; HRMS cald for $\mathrm{C}_{17} \mathrm{H}_{26} \mathrm{~N}_{2} \mathrm{O}_{7}$ $\left[\mathrm{MNa}^{+}\right]: 393.1638$ Found: 393.1628.<smiles>C[C@@H](C(=O)O)[C@H](OC/C=C/CCC(=O)N(C)C(=O)CN(C(=O)OCc1ccccc1)C(=O)OCc1ccccc1)c1ccccc1</smiles>

$3 g$

3g: ${ }^{1} \mathrm{H}$ NMR (300 $\mathbf{M H z}, \mathbf{C D C l}_{3}$ ): Observed spectrum of $3 \mathrm{~g}$ is a complex mixture of double bond isomers and rotamers. Approximate $Z / E$ ratio extracted from spectra obtained in toluene at elevated temperatures. See spectra provided.

${ }^{13} \mathrm{C}$ NMR (75 $\mathrm{MHz}, \mathrm{CDCl}_{3}$ ): Mixture of double bond isomers and rotamers.

${ }^{1} \mathbf{H}$ NMR (500 MHz, $\left.\mathbf{C}_{7} \mathbf{D}_{8}\right)$ : Mixture of double bond isomers $(Z: E=3: 2)$ and rotamers. See provided spectra from variable temperature experiments.

HRMS calcd for $\mathrm{C}_{34} \mathrm{H}_{37} \mathrm{~N}_{3} \mathrm{O}_{7}$ [MNa ${ }^{+}$]: 622.2529 Found: 622.2507 .

\section{Representative procedure for the formation of cyclic hydrazine derivatives $3 \mathbf{h} \&$ $3 \mathbf{i}$ via RCM}

Enyne $2 \mathbf{h}(49 \mathrm{mg}, 0.12 \mathrm{mmol})$ was dissolved in $\mathrm{CH}_{2} \mathrm{Cl}_{2}(0.002 \mathrm{M})$ in a $250 \mathrm{~mL}$ round bottom flask and the solution was flushed with $\mathrm{N}_{2}$ for approximately 5 minutes. The flask was fitted with a reflux condenser and Grubbs second generation catalyst $(5 \mathrm{~mol} \%$ dissolved in $1 \mathrm{~mL}$ of $\mathrm{CH}_{2} \mathrm{Cl}_{2}$ ) was added. A steady flow of ethylene was bubbled through the solution and the reaction was refluxed for 5 hours. The solvent was removed in vacuo and the residue was purified by silica gel chromatography (3:1 hexane:ethyl acetate), yielding approximately $1: 1$ mixture of $\mathbf{3 h}$ and ethylene cross metathesis product 3h' (24 mg, 47 \%), and small amount of recovered starting material.

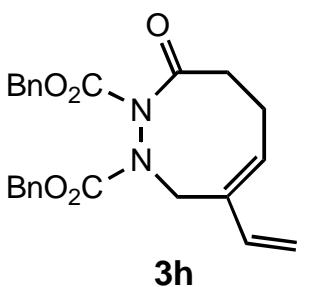


3h: ${ }^{1} \mathbf{H}$ NMR (300 MHz, $\mathbf{C D C l}_{3}$ ) major rotamer: $\delta 7.23-7.39(\mathrm{~m}, 10 \mathrm{H}), 6.23(\mathrm{dd}, J=17.3$, $11.2,1 \mathrm{H}), 5.93(\mathrm{t}, J=7.9,1 \mathrm{H}), 4.88-5.25(\mathrm{~m}, 4 \mathrm{H}$, mixture of rotamers), $5.15(\mathrm{~d}, J=15.7,1 \mathrm{H})$, $4.90(\mathrm{dd}, J=10.5,1.9,1 \mathrm{H}), 4.81(\mathrm{dd}, J=15.7,1.9,1 \mathrm{H}), 4.18(\mathrm{~d}, J=15.7,1 \mathrm{H}), 2.77(\mathrm{~m}, 1 \mathrm{H})$, 2.48-2.69 (m, 2H), $2.20(\mathrm{~m}, 1 \mathrm{H})$; minor rotamer: $\delta 4.73(\mathrm{dd}, J=15.7,1.9,1 \mathrm{H}), 4.25(\mathrm{~d}, J=$ $15.7,1 \mathrm{H}), 2.91(\mathrm{~m}, 1 \mathrm{H}), 2.25(\mathrm{~m}, 1 \mathrm{H})$.

${ }^{13}$ C NMR (75 MHz, $\mathbf{C D C l}_{3}$ ) major rotamer: $\delta$ 172.53, 155.14, 150.77, 137.69, 135.37, 130.89, $128.62,128.22,128.15,113.00,69.01,68.61,45.95,37.32,22.38$; minor rotamer: $\delta 155.27$, $154.99,151.15,150.54,134.64,134.49,134.36,131.12,128.68,128.57,128.54,128.38,127.92$, 69.13, 46.72, 22.53; HRMS calcd for $\mathrm{C}_{24} \mathrm{H}_{24} \mathrm{~N}_{2} \mathrm{O}_{5}\left[\mathrm{MNa}^{+}\right]$: 443.1583 Found: 443.1579 .

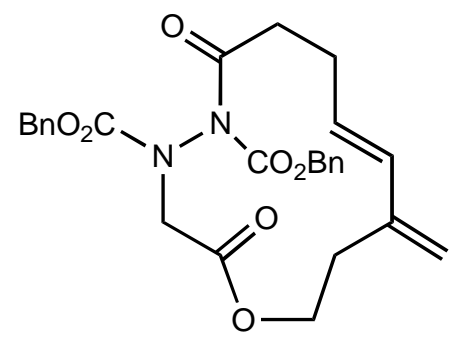

3i

3i: ${ }^{1}$ H NMR (300 MHz, $\left.\mathbf{C D C l}_{3}\right) \delta$ 7.13-7.40 (m, 10H), 6.10 (d, $\left.J=15.2,1 \mathrm{H}\right), 5.68(\mathrm{dt}, J=15.2$, 8.0, 1H), 5.27 (d, $J=0.8,1 \mathrm{H}), 5.01,5.07,5.10,5.17(\mathrm{~d}, J=12.5,4 \mathrm{H}), 4.93(\mathrm{~d}, J=0.8,1 \mathrm{H}), 4.59$ $(\mathrm{d}, J=17.1,1 \mathrm{H}), 4.28(\mathrm{~m}, 1 \mathrm{H}$, mixture of rotamers $), 4.14(\mathrm{~d}, J=17.1,1 \mathrm{H}), 4.04(\mathrm{~m}, 1 \mathrm{H}$, mixture of rotamers), $3.73(\mathrm{~m}, 1 \mathrm{H}$, mixture of rotamers), $2.58(\mathrm{~m}, 4 \mathrm{H}$, mixture of rotamers), $2.34(\mathrm{~m}, 1 \mathrm{H}$, mixture of rotamers); minor rotamer: $\delta 6.09(\mathrm{~d}, J=15.2,1 \mathrm{H}), 5.67(\mathrm{dt}, J=15.2,8.0,1 \mathrm{H}), 5.16$, $5.21(\mathrm{~d}, J=12.54 .91,2 \mathrm{H}),(\mathrm{d}, J=0.8,1 \mathrm{H}), 4.45(\mathrm{~d}, J=17.2,1 \mathrm{H}), 4.17(\mathrm{~d}, J=17.2,1 \mathrm{H})$.

${ }^{13}$ C NMR (75 MHz, CDCl $)$ major rotamer: $\delta$ 172.02, 166.99, 154.87, 153.17, 143.54, 135.29, $134.46,134.19,128.41,128.38,128.25,128.00,127.43,126.88,115.34,69.06,68.25,66.54$, 49.34, 34.99, 30.84, 29.51; minor rotamer: $\delta 166.78,154.36,153.07,143.63,135.15,134.65$, $134.49,128.47,128.35,128.18,127.97,127.85,127.78,127.21,115.10,69.14,68.70,66.61$, 50.19, 35.11, 31.01, 29.58, 28.26; HRMS calcd for $\mathrm{C}_{27} \mathrm{H}_{28} \mathrm{~N}_{2} \mathrm{O}_{7}\left[\mathrm{MNa}^{+}\right]$: 515.1794 Found: 515.1780 .

Synthesis of 9 (racemic) 
To a solution of 3a $(177 \mathrm{mg}, 0.42 \mathrm{mmol})$ in $5 \mathrm{~mL}$ of acetone- $\mathrm{H}_{2} \mathrm{O}-\mathrm{CH}_{2} \mathrm{Cl}_{2}$ (1:1:1), was added excess Oxone ${ }^{\circledR}(336 \mathrm{mg})$ and $\mathrm{NaHCO}_{3}(223 \mathrm{mg})$ at $0{ }^{\circ} \mathrm{C}$. The reaction was stirred at $0{ }^{\circ} \mathrm{C}$ for 6 hours then gradually warmed up to room temperature and stirred for an additional 6 hours. The reaction was quenched with saturated $\mathrm{NaHSO}_{3}$ and extracted with $1: 1$ ethyl acetate:hexane $(2 \times$ $3.0 \mathrm{~mL}$ ). The combined organic layer was dried over $\mathrm{MgSO}_{4}$ and concentrated in vacuo, and the residue was purified by silica gel chromatography (2:1 hexane:ethyl acetate) to afford epoxide 3a-1 as a colorless oil (160 mg, 87 \%). Compound 3a-1 (80 mg, $0.18 \mathrm{mmol}$ ) was then dissolved in $3 \mathrm{~mL}$ of methanol, and to this solution was added $\mathrm{Pd} / \mathrm{C}(10 \mathrm{wt} \%, 14 \mathrm{mg})$. The reaction mixture was maintained under a blanket of $\mathrm{H}_{2}$ (47 psi) for 16 hours, then filtered through a layer of celite/silica gel, and washed with ethyl acetate to afford $\mathbf{9}$ as a white crystalline solid (30 mg, $97 \%)$.

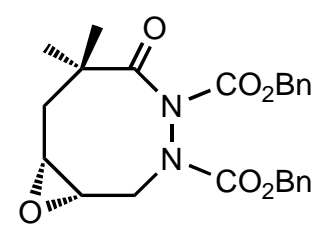

3a-1

3a-1: ${ }^{1}$ H NMR (300 MHz, $\mathbf{C D C l}_{3}$ ) major rotamer: $\delta 7.33(\mathrm{~m}, 10 \mathrm{H}), 5.22,5.13,5.12,4.98(\mathrm{~d}, J$ $=12.2,4 \mathrm{H}), 4.20(\mathrm{dd}, J=14.7,3.2,1 \mathrm{H}), 3.97(\mathrm{dd}, J=14.6,5.7,1 \mathrm{H}), 3.18(\mathrm{dt} J=11.2,3.8,1 \mathrm{H})$, $2.97(\mathrm{dt}, J=5.5,3.4,1 \mathrm{H}), 2.20(\mathrm{dd}, J=13.6,3.71 \mathrm{H}), 1.75(\mathrm{dd}, J=13.6,11.5,1 \mathrm{H}), 1.37(\mathrm{~s}, 3 \mathrm{H})$, $1.09(\mathrm{~s}, 3 \mathrm{H})$; minor rotamer: $\delta 5.15-5.30(\mathrm{~m}, 4 \mathrm{H}), 4.03(\mathrm{dd}, J=14.6,5.7,1 \mathrm{H}), 1.37(\mathrm{~s}, 3 \mathrm{H})$, $1.17(\mathrm{~s}, 3 \mathrm{H})$.

${ }^{13}$ C NMR (75 MHz, $\mathbf{C D C l}_{3}$ ) major rotamer: $\delta$ 176.33, 155.09, 151.61, 134.61, 134.35, 128.49, $128.36,128.17,128.11,127.96,127.84,69.22$, 69.60, 52.64, 49.78, 49.38, 43.96, 40.23, 28.25, 27.17; minor rotamer: $\delta 176.47,155.21,151.93,134.96,134.53,128.44,128.26,128.23$, 128.02, 69.34, 68.90, 52.96, 50.12, 44.05, 40.55, 28.16, 27.01; HRMS calcd for $\mathrm{C}_{24} \mathrm{H}_{26} \mathrm{~N}_{2} \mathrm{O}_{6}$ [MNa ${ }^{+}$]: 461.1689 Found: 461.1693.

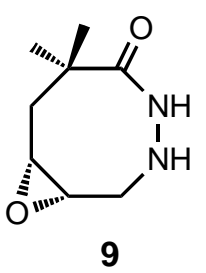


9: ${ }^{1}$ H NMR (300 MHz, $\mathbf{C D C l}_{3}$ ) $\delta 7.27$ (br s, 1H), 3.74 (br s, 1H), 3.52 (dd, $\left.J=14.2,4.7,1 \mathrm{H}\right)$, $3.40(\mathrm{~d}, J=14.3,1 \mathrm{H}), 3.26(\mathrm{dt}, J=10.5,4.5,1 \mathrm{H}), 3.03($ br m, 1H), 2.71 (dd, $J=12.6,5.4,1 \mathrm{H})$, $2.19(\mathrm{dd}, J=13,4.81 \mathrm{H}), 1.36(\mathrm{~s}, 3 \mathrm{H}), 1.33(\mathrm{~s}, 3 \mathrm{H})$.

${ }^{13}$ C NMR (75 MHz, $\mathbf{C D C l}_{3}$ ) $\delta 181.67,54.51,52.17,51.87,41.18,41.06,28.15,26.85$; HRMS calcd for $\mathrm{C}_{8} \mathrm{H}_{14} \mathrm{~N}_{2} \mathrm{O}_{2}\left[\mathrm{MNa}^{+}\right]$: 193.0953 Found: 193.0949 .

\section{Synthesis of $\mathbf{1 0}$}

To a solution of 9 (200 mg, $1.18 \mathrm{mmol})$ in $\mathrm{CH}_{2} \mathrm{Cl}_{2}(5 \mathrm{~mL})$ was added 4-pentenoic acid $(0.28 \mathrm{~mL}$, $2.36 \mathrm{mmol})$, EDC (500mg, $2.36 \mathrm{mmol})$, and DMAP (5 mg, $3 \mathrm{~mol} \%$ ). The reaction was allowed to stir at room temperature for 24 hours, and then the solution was concentrated in vacuo and the residue was purified by silica gel chromatography (3:1 hexnae:ethyl acetate) to yield 3a-2 as a yellowish oil (155 mg, 54 \%). The acylated compound 3a-2 (155mg, $0.62 \mathrm{mmol}$ ) was then dissolved in DMF $(2.0 \mathrm{~mL})$, and to this solution was added $\mathrm{Cs}_{2} \mathrm{CO}_{3}(240 \mathrm{mg}, 0.75 \mathrm{mmol})$ followed by allyl bromide $(0.02 \mathrm{~mL}, 1.24 \mathrm{mmol})$. The solution was stirred at room temperature for 2 hours, then diluted with $\mathrm{CH}_{2} \mathrm{Cl}_{2}(2.0 \mathrm{~mL})$ and washed water $(5 \times 2.0 \mathrm{~mL})$ to remove residual DMF. The combined organic layer was then dried over $\mathrm{MgSO}_{4}$, concentrated in vacuo, and the residue was purifiedy by silica gel chromatography (3:1 hexane:ethyl acetate) to yield 10 as colorless oil (140 mg, 78 \%). [EDC: 1-[3-(Dimethylaminopropyl]-3-ethylcarbodiimide $\cdot \mathrm{HCl}]$

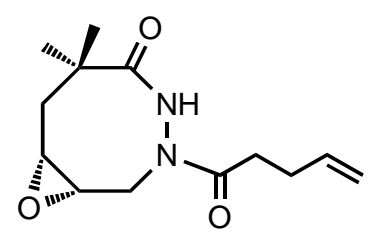

$3 a-2$

3a-2: ${ }^{1} \mathbf{H}$ NMR (300 MHz, $\mathbf{C D C l}_{3}$ ) $\delta 8.21$ (br s, 1H), 7.99 (br s, 1H; minor rotamer), 5.83 (dddd, $J=17.0,10.4,6.6,6.5,1 \mathrm{H}), 5.07$ (dd, $J=17.0,1.4,1 \mathrm{H}), 5.02(\mathrm{dd}, J=10.4,1.4,1 \mathrm{H}), 4.67$ (br m, 1H), 3.39 (br d, $J=16,1 \mathrm{H}$; minor rotamer), 3.55 (br m, 1H), 3.25 (m, 1H), 3.14 (m, 1H), $2.56(\mathrm{~m}, 2 \mathrm{H}), 2.40(\mathrm{~m}, 2 \mathrm{H}), 2.18(\mathrm{dd}, J=14.0,4.4,1 \mathrm{H}), 1.85(\mathrm{dd}, J=14.0,10.7,1 \mathrm{H}), 1.38(\mathrm{~s}$, $3 \mathrm{H}), 1.26(\mathrm{~s}, 3 \mathrm{H})$ 
${ }^{13} \mathrm{C}$ NMR (75 MHz, $\mathbf{C D C l}_{3}$ ) mixture of rotamers: $\delta$ 182.39, 181.22, 177.46, 136.99, 136.89, 136.76, 116.21, 116.04, 115.79, 53.67, 53.31, 51.23, 48.86, 45.96, 41.68, 38.95, 33.47, 32.74, 31.94, 28.98, 28.85, 28.79, 27.33; HRMS calcd for [MNa ${ }^{+}$]: 275.1372 Found: 275.1369.

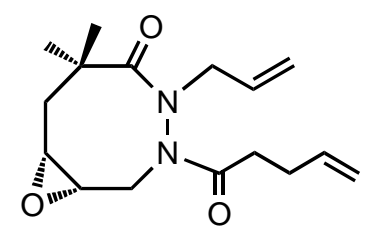

10

10: ${ }^{1} \mathbf{H}$ NMR (300 MHz, $\left.\mathbf{C D C l}_{3}\right)$ major rotamer: $\delta 5.99(\mathrm{~m}, 1 \mathrm{H}), 5.82(\mathrm{~m}, 1 \mathrm{H}), 5.28(\mathrm{~m}, 2 \mathrm{H})$, $5.06(\mathrm{~m}, 2 \mathrm{H}), 4.55(\mathrm{dd}, J=14.6,5.8,1 \mathrm{H}), 4.46(\mathrm{dd}, J=15.2,3.5,1 \mathrm{H}), 3.69(\mathrm{dd}, J=14.8,7.8$, $1 \mathrm{H}), 3.21(\mathrm{dt}, J=10.7,4.0,1 \mathrm{H}), 3.08(\mathrm{~m}, 1 \mathrm{H}), 2.41(\mathrm{~m}, 5 \mathrm{H}), 2.18(\mathrm{dd}, J=13.7,3.7,1 \mathrm{H}), 1.61$ $(\mathrm{dd}, J=14.3,10.9,1 \mathrm{H}), 1.42(\mathrm{~s}, 3 \mathrm{H}), 1.23(\mathrm{~s}, 3 \mathrm{H})$; minor rotamer: $\delta 4.12(\mathrm{dd} J=15.2,2.0$, 1H), 4.02 (dd, $J=15.2,4.7,1 \mathrm{H}), 3.86$ (dd, $J=14.9,7.8,1 \mathrm{H}), 3.74(\mathrm{dd}, J=14.9,5.6,1 \mathrm{H}), 3.28$ $(\mathrm{dt}, J=10.3,4.4,1 \mathrm{H}), 2.21(\mathrm{dd}, J=13.7,4.8,1 \mathrm{H}), 1.70(\mathrm{dd}, J=13.4,10.3,1 \mathrm{H}), 1.18(\mathrm{~s}, 3 \mathrm{H})$.

${ }^{13} \mathbf{C}$ NMR (75 MHz, $\mathbf{C D C l}_{3}$ ) mixture of rotamers: $\delta$ 178.27, 177.99, 175.31, 171.79, 136.85, $136.74,133.53,132.28,120.37,119.52,116.27,54.72$, 54.05, 52.96, 52.23, 51.67, 50.46, 50.37, 47.27, 42.41, 40.22, 39.75, 33.14, 32.67, 29.24, 29.11, 28.85, 28.68, 28.63; HRMS calcd for $\left[\mathrm{MNa}^{+}\right]: 315.1685$ Found: 315.1698.

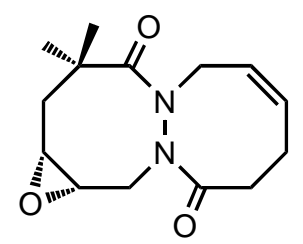

11

11: ${ }^{1}$ H NMR (300 MHz, $\left.\mathbf{C D C l}_{3}\right) \delta 5.98(\mathrm{~m}, 1 \mathrm{H}), 5.64(\mathrm{~m}, 1 \mathrm{H}), 4.93(\mathrm{dt}, J=16.2,2.1 \mathrm{~Hz}, 1 \mathrm{H})$, $4.45(\mathrm{dd}, J=15.5,3.5 \mathrm{~Hz}, 1 \mathrm{H}), 3.62(\mathrm{dd}, J=16,6.6,1 \mathrm{H}), 3.51$ (dd, $J=15.4,5.8,1 \mathrm{H}), 3.18$ (dt, $J$ $=11.0,4.0), 3.02(\mathrm{dt}, J=5.8,3.4), 2.96(\mathrm{t}, J=6.7), 2.56(\mathrm{~m}, 2 \mathrm{H}), 2.27(\mathrm{~m}, 1 \mathrm{H}), 2.19(\mathrm{dd} J=$ 13.9, 3.8, 1H), $1.68(\mathrm{ddd}, J=13.9,10.9,0.7,1 \mathrm{H}), 1.41$ (s, 3H), 1.27 (s, 3H).

${ }^{13} \mathrm{C}$ NMR (75 MHz, $\left.\mathbf{C D C l}_{3}\right) \delta$ 178.72, 176.81, 134.23, 123.27, 53.05, 49.73, 46.38, 45.69, 42.41, 39.81, 35.26, 28.75, 28.22, 23.40; HRMS calcd for [ $\left.\mathrm{MNa}^{+}\right]$: 287.1372 Found: 287.1382. 
Synthesis of 12 from 11 via reductive $\mathrm{N}-\mathrm{N}$ bond cleavage

Bicycle 11 (102 mg, $0.39 \mathrm{mmol})$ was dissolved in THF $(1.5 \mathrm{~mL})$ in a 3-neck $25 \mathrm{~mL}$ round bottom flask. The flask was fitted with a cold finger and $\mathrm{NH}_{3}(10 \mathrm{~mL})$ was condensed in to the reaction flask at $-78{ }^{\circ} \mathrm{C}$, and sodium was added until solution maintained a persistent deep blue color. The reaction was stirred at $-78{ }^{\circ} \mathrm{C}$ for 2.5 hours then quenched with excess solid $\mathrm{NH}_{4} \mathrm{Cl}$ (approx. 10 eq.), and the solution was gradually warmed up to room temperature to evaporate the ammonia. The aqueous THF solution was then quenched with 1:1 $\mathrm{MeOH}: \mathrm{H}_{2} \mathrm{O}$, and the solution was extracted with ethyl acetate $(3 \times 2.0 \mathrm{~mL})$. The combined organic layer was dried over $\mathrm{MgSO}_{4}$ and concentrated in vacuo to yield 12 as a white crystalline solid (87 mg, 85 \%).<smiles>CC1(C)CCCC(=O)NC/C=C\CCC(=O)N/C=C/[C@H](O)C1</smiles>

12

12: ${ }^{1}$ H NMR (300 MHz, CD CDD) $\delta 6.73(\mathrm{~d}, J=14.5,1 \mathrm{H}), 5.69(\mathrm{ddd}, J=11.2,11.2,4.0,1 \mathrm{H})$, 5.57 (ddd, $J=11.2,11.2,3.7,1 \mathrm{H}), 4.98$ (dd, 14.5, 9.7, 1H), 4.08 (m, 2H), 2.78 (br m, 1H), 2.57 $(\mathrm{dt}, J=13.8,4.8,1 \mathrm{H}), 2.17(\mathrm{~m}, 4 \mathrm{H}), 1.54(\mathrm{dd}, J=12.9,3.9,1 \mathrm{H}), 1.21(\mathrm{~s}, 3 \mathrm{H}), 1.12(\mathrm{~s}, 3 \mathrm{H})$. ${ }^{13}$ C NMR (75 MHz, CD ${ }_{3}$ OD) $\delta 180.01,173.10,131.73,126.68,125.28,114.88,70.47,41.26$, 39.48, 37.48, 30.81, 25.36, 21.92; HRMS calcd for [ $\left.\mathrm{MH}^{+}\right]$: 267.1709 Found: 267.1699. 

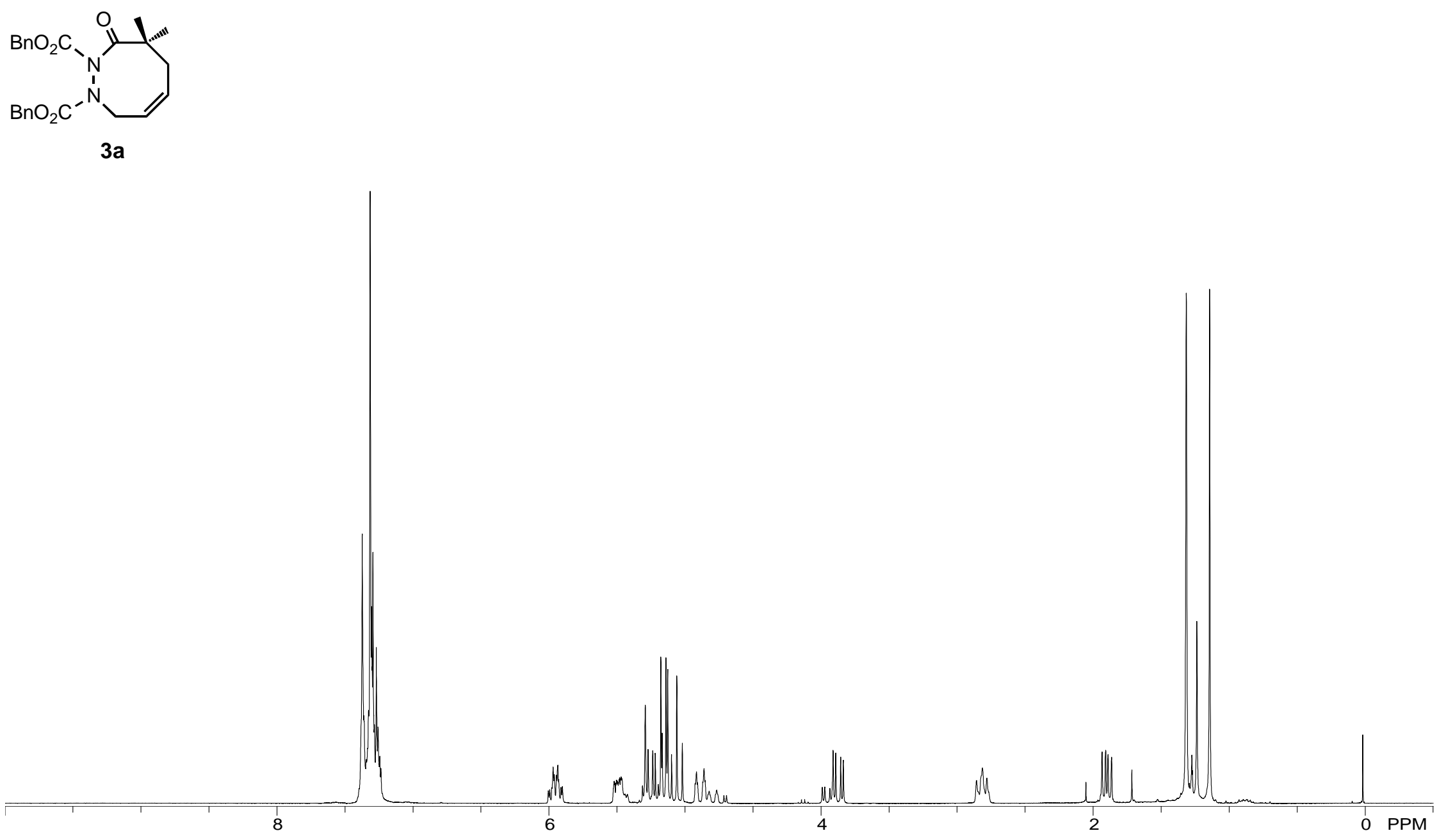

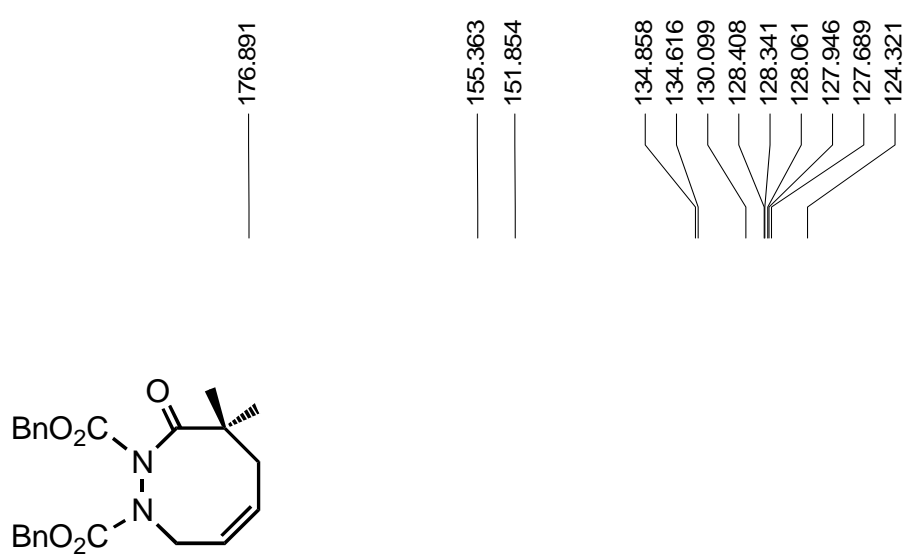

$\begin{array}{lll}\hat{L} & 0 \\ \infty & 0 \\ \infty & 0 \\ \infty & \infty \\ 0 & 0 & 0\end{array}$

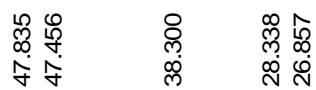

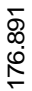

\section{$3 \mathbf{a}$}




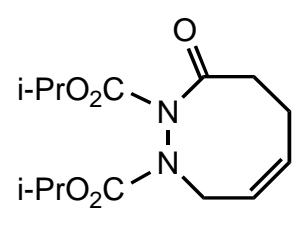

3b

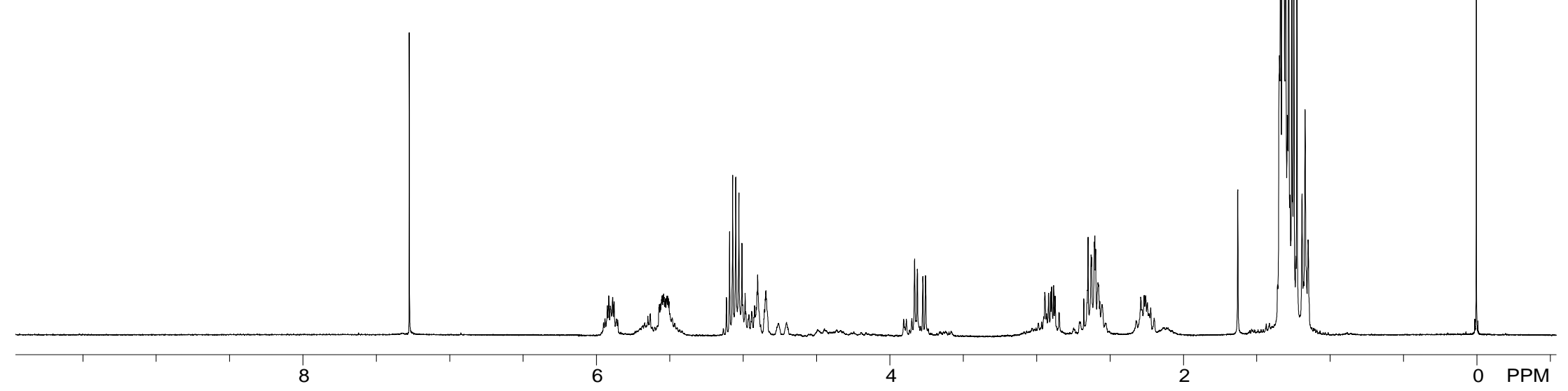



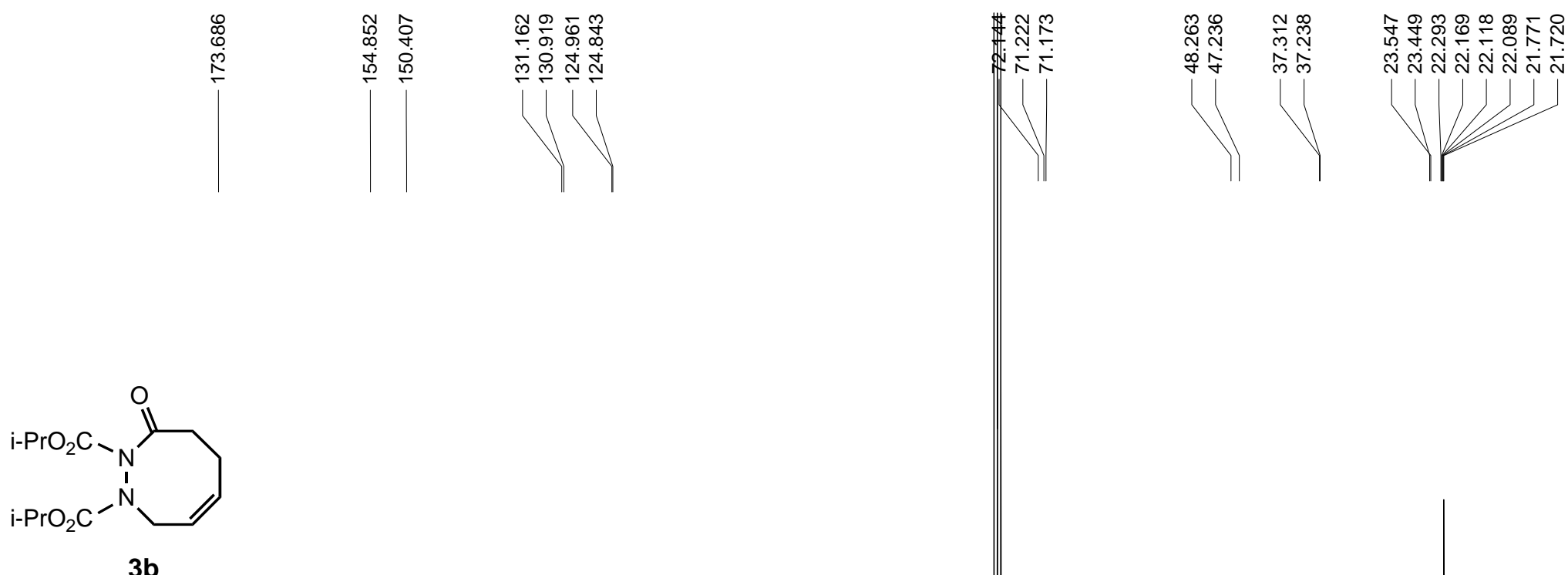

$3 b$

\&

ระ 


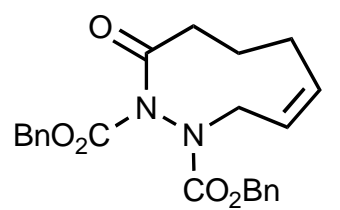

$3 c$

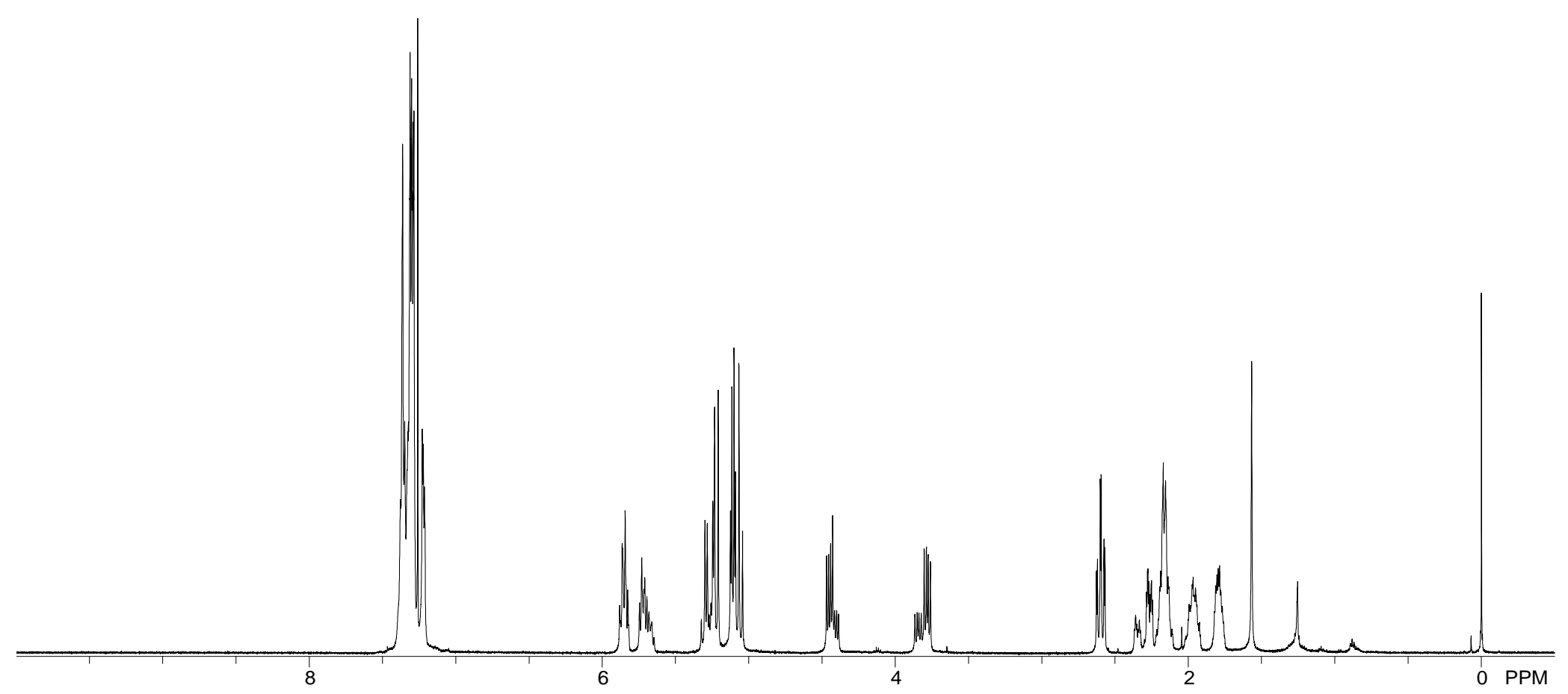


กิ

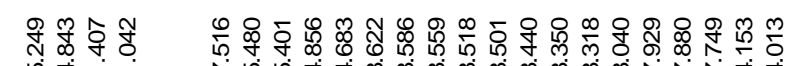

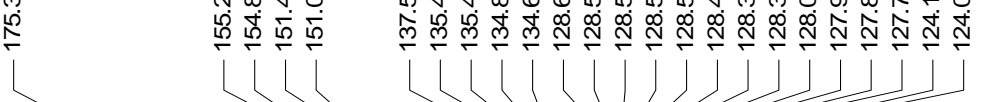

둥용으뭉

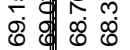

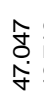

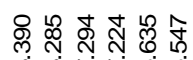

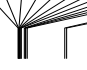

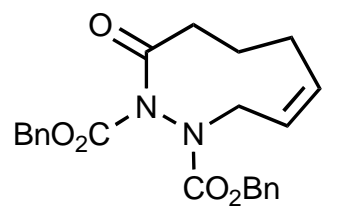

$3 c$

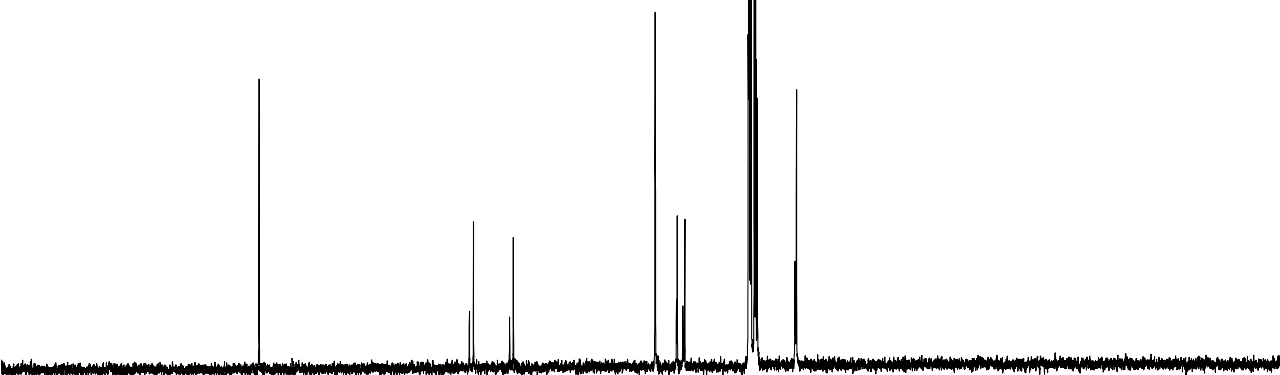




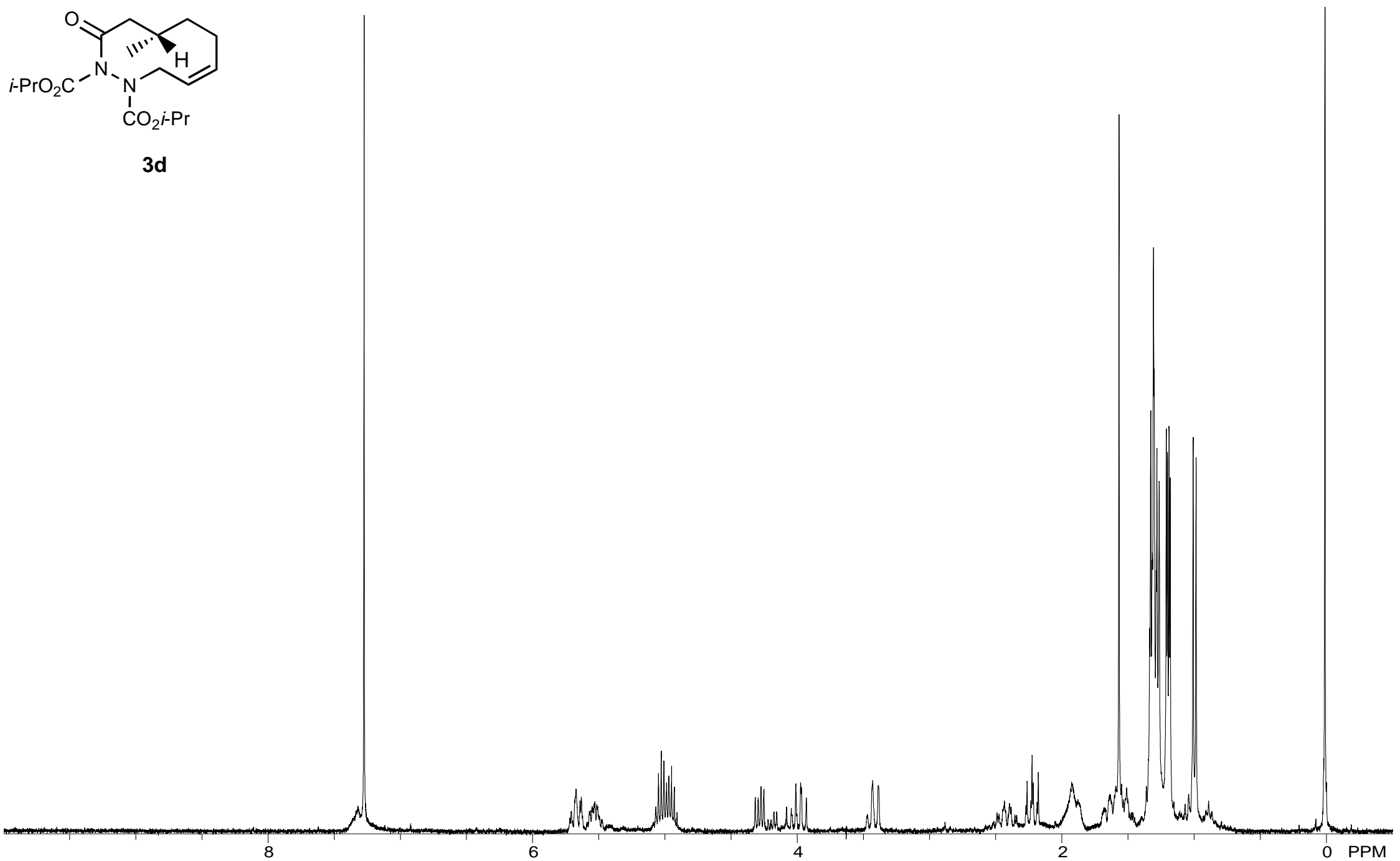




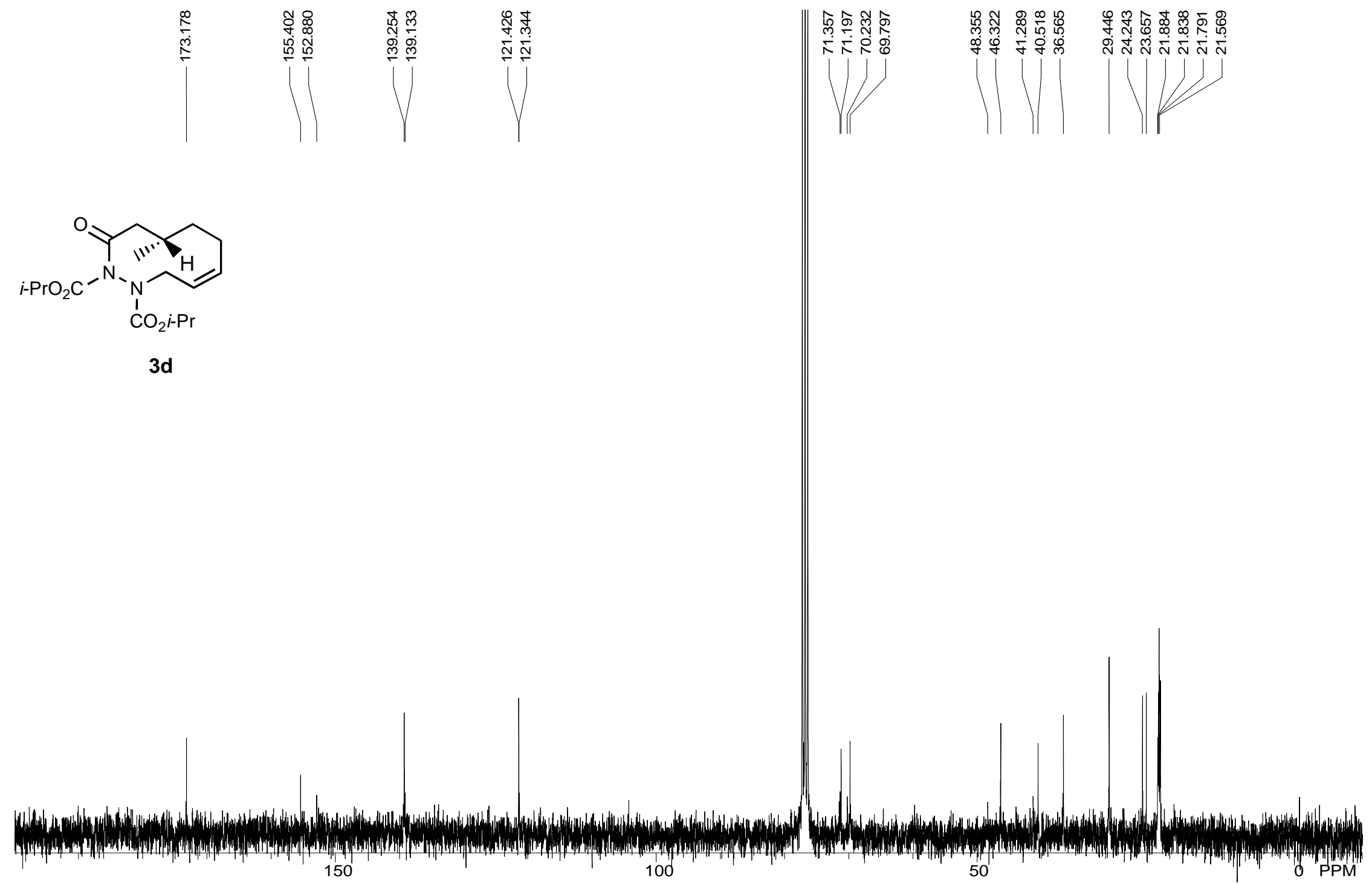



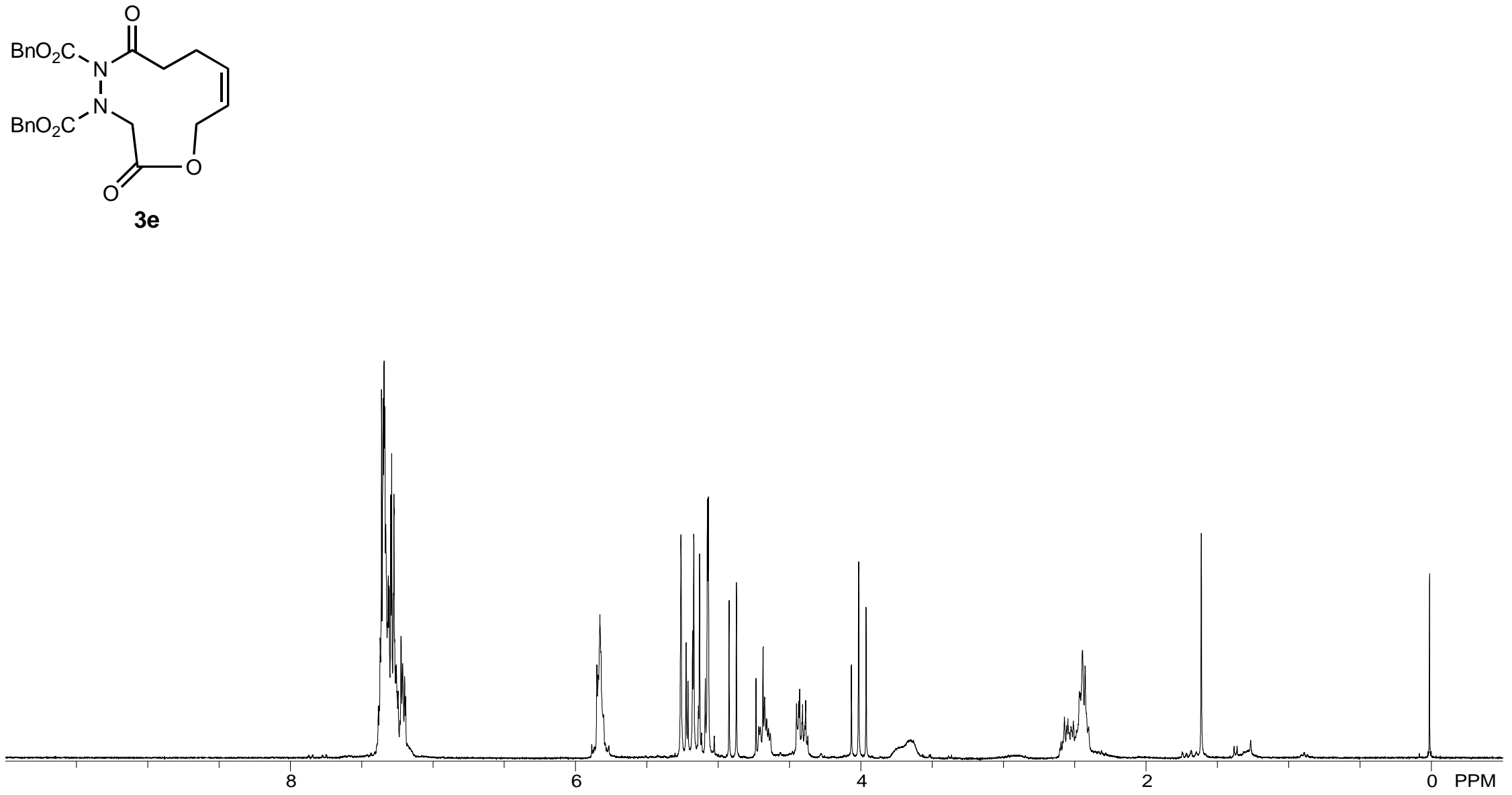


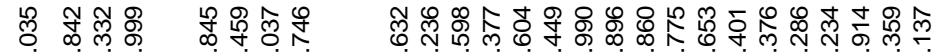

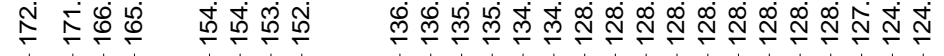

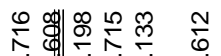

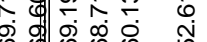

今ิ

1
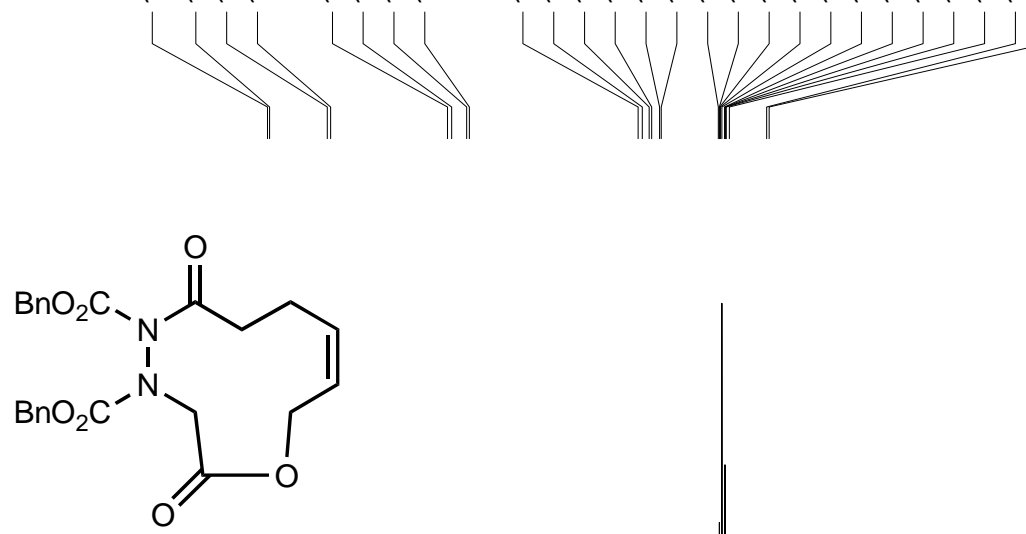

$3 e$

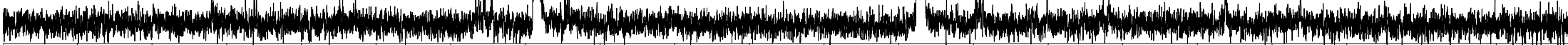




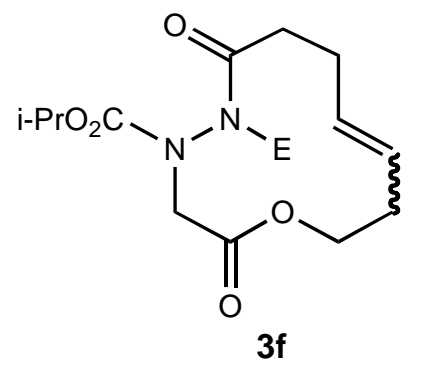




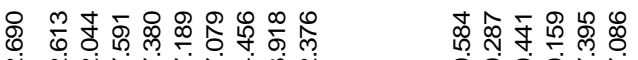

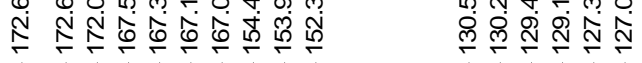<smiles>CCC=CCOC(=O)CN1CCCCCCCCCCCCCCCCCC1</smiles>

$3 f$

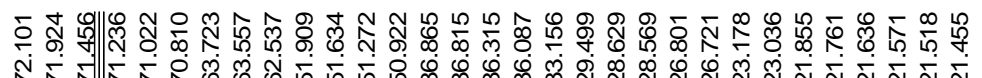




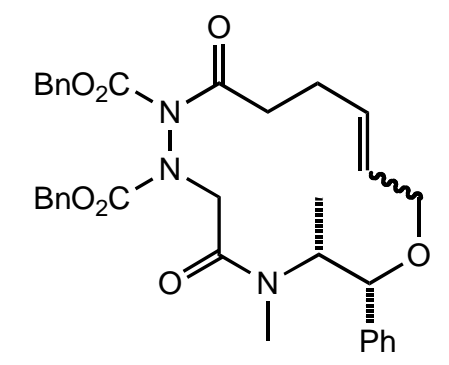

$3 g$

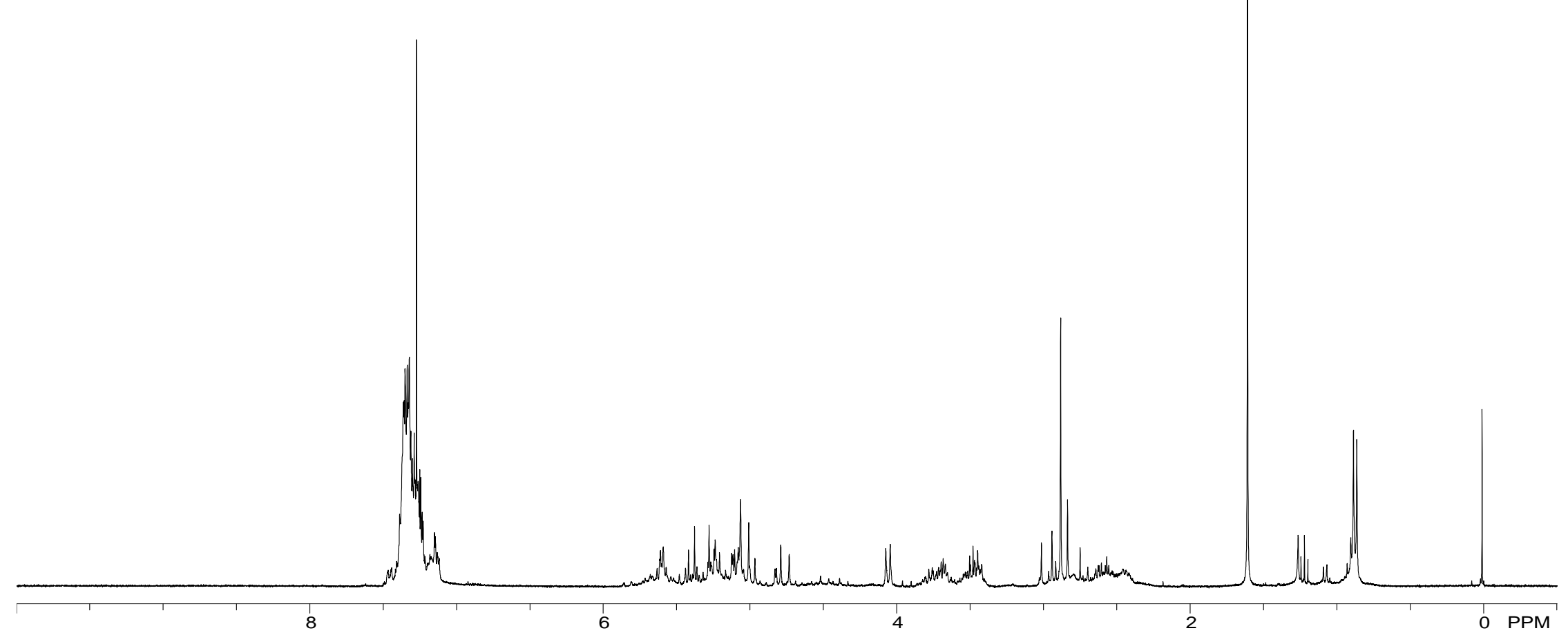




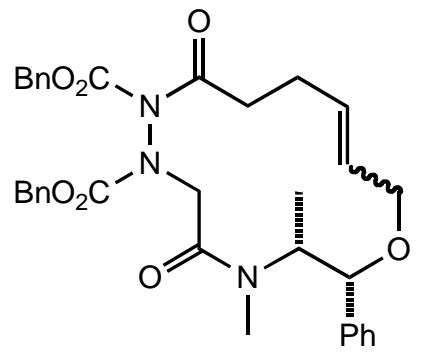

$3 g$

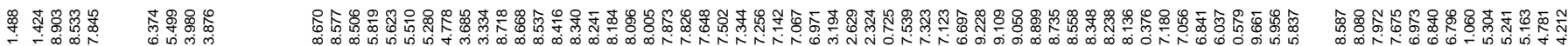
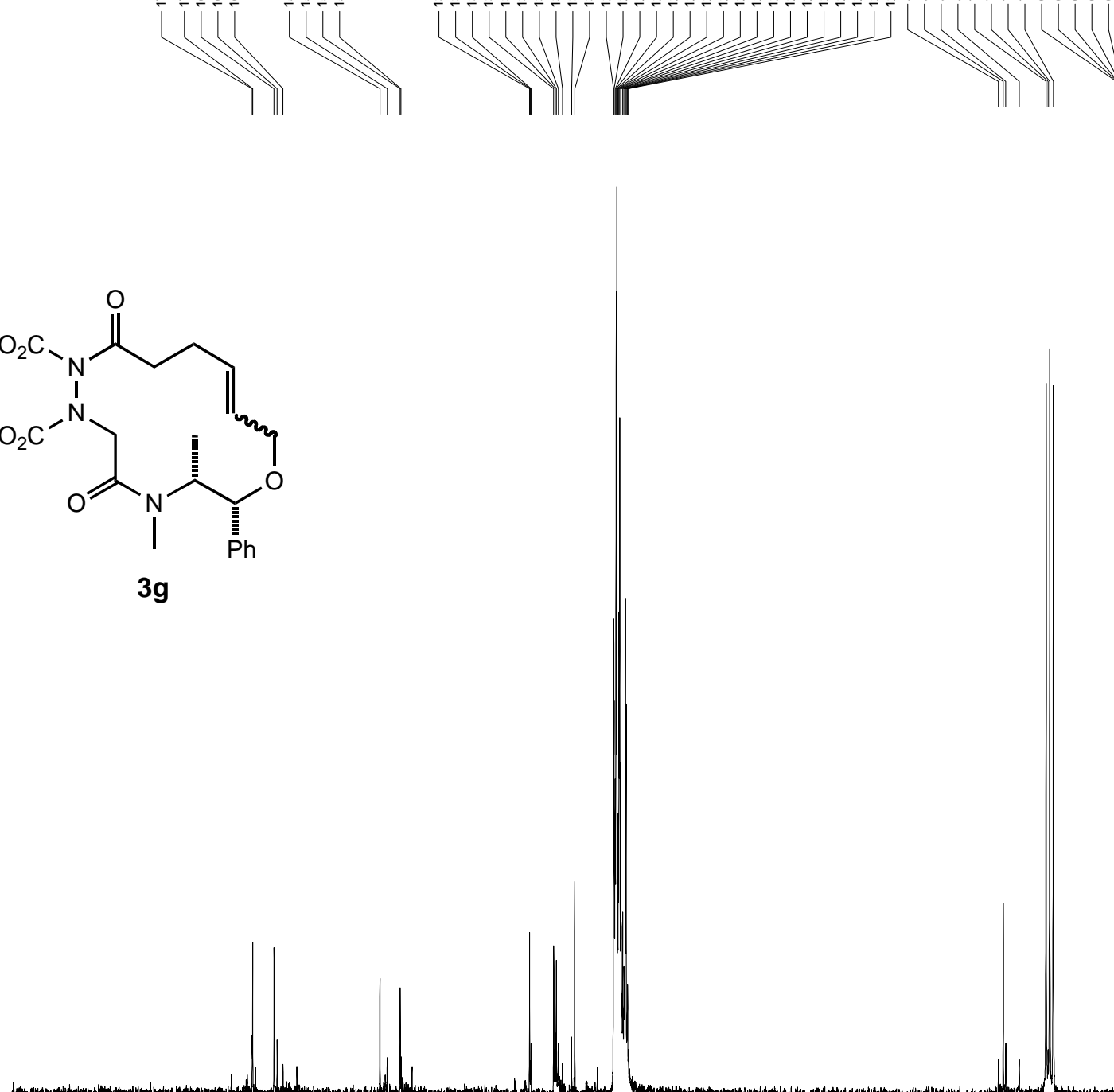

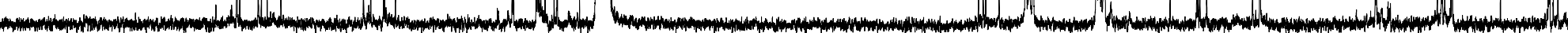




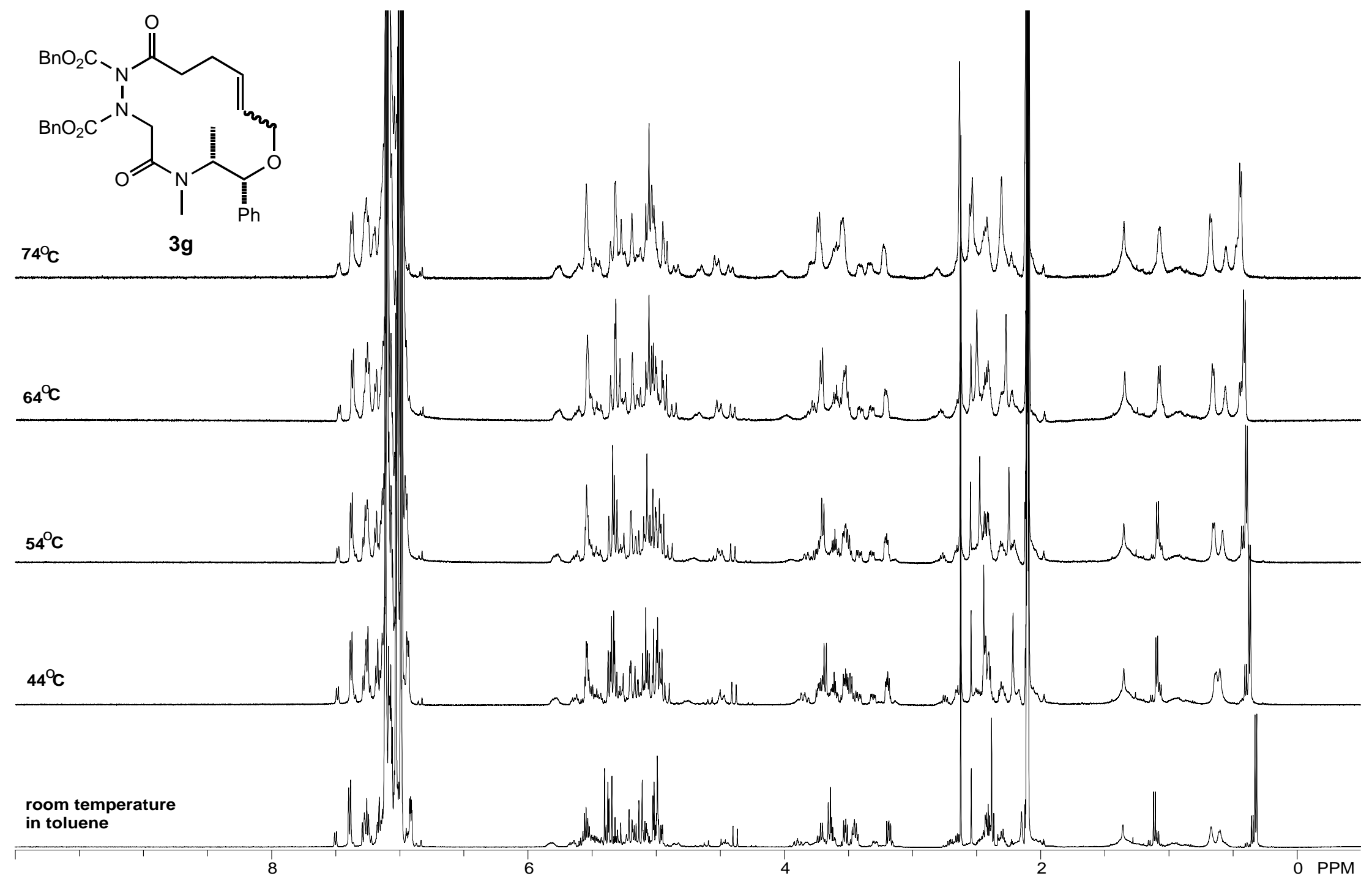




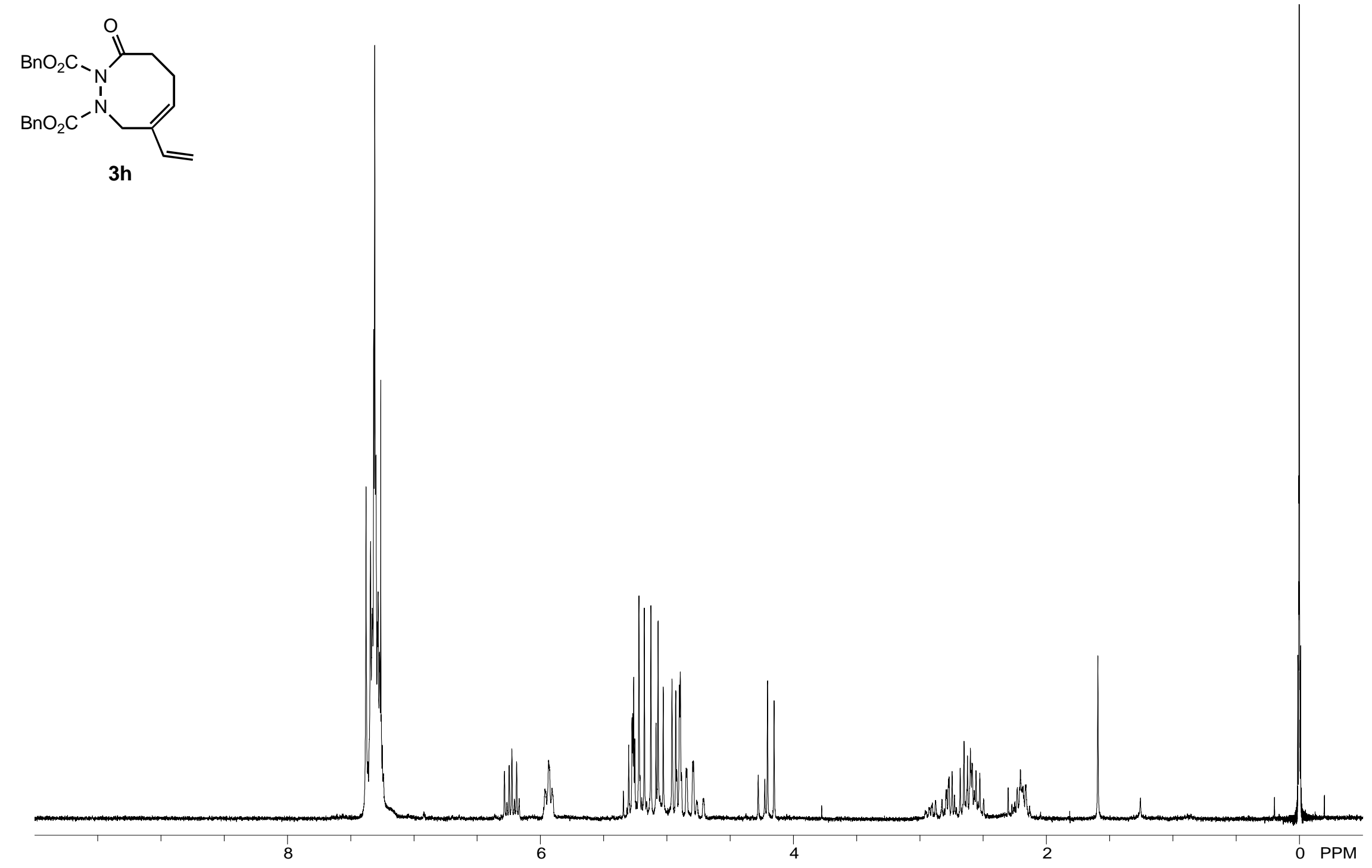




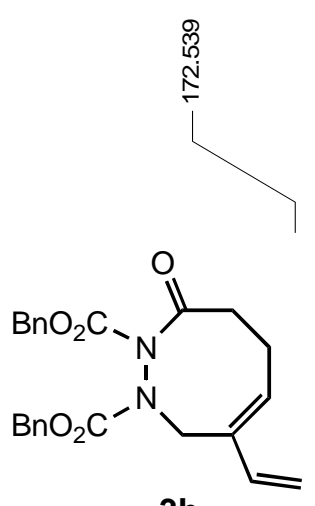

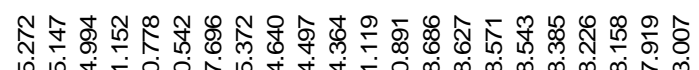

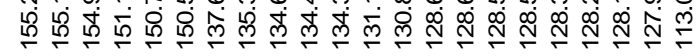

$3 \mathrm{~h}$

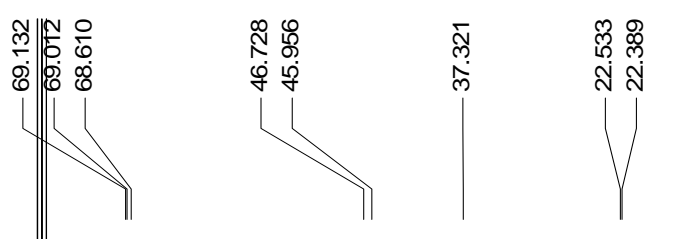

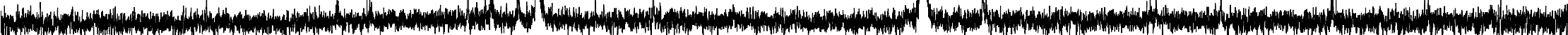



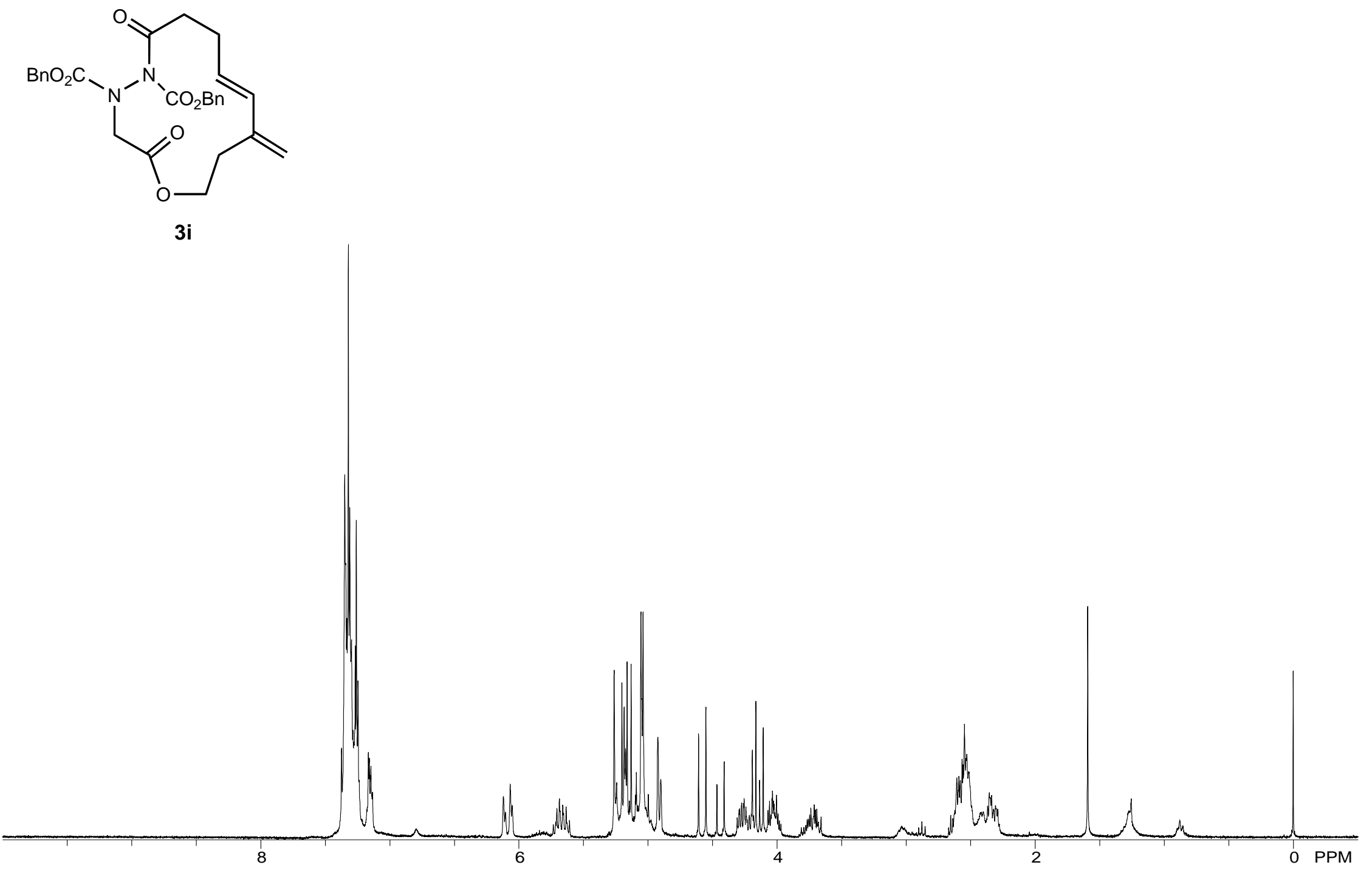


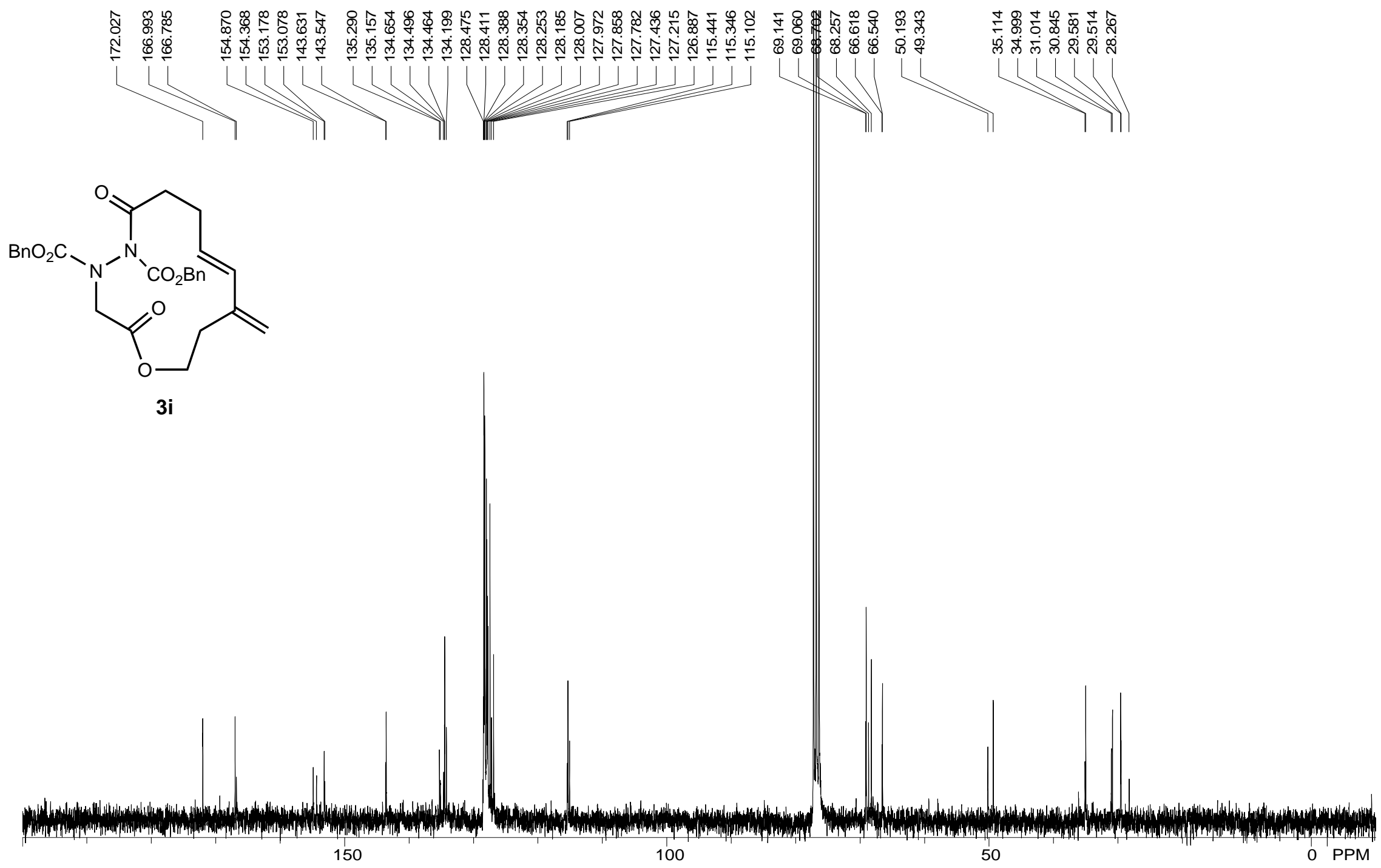



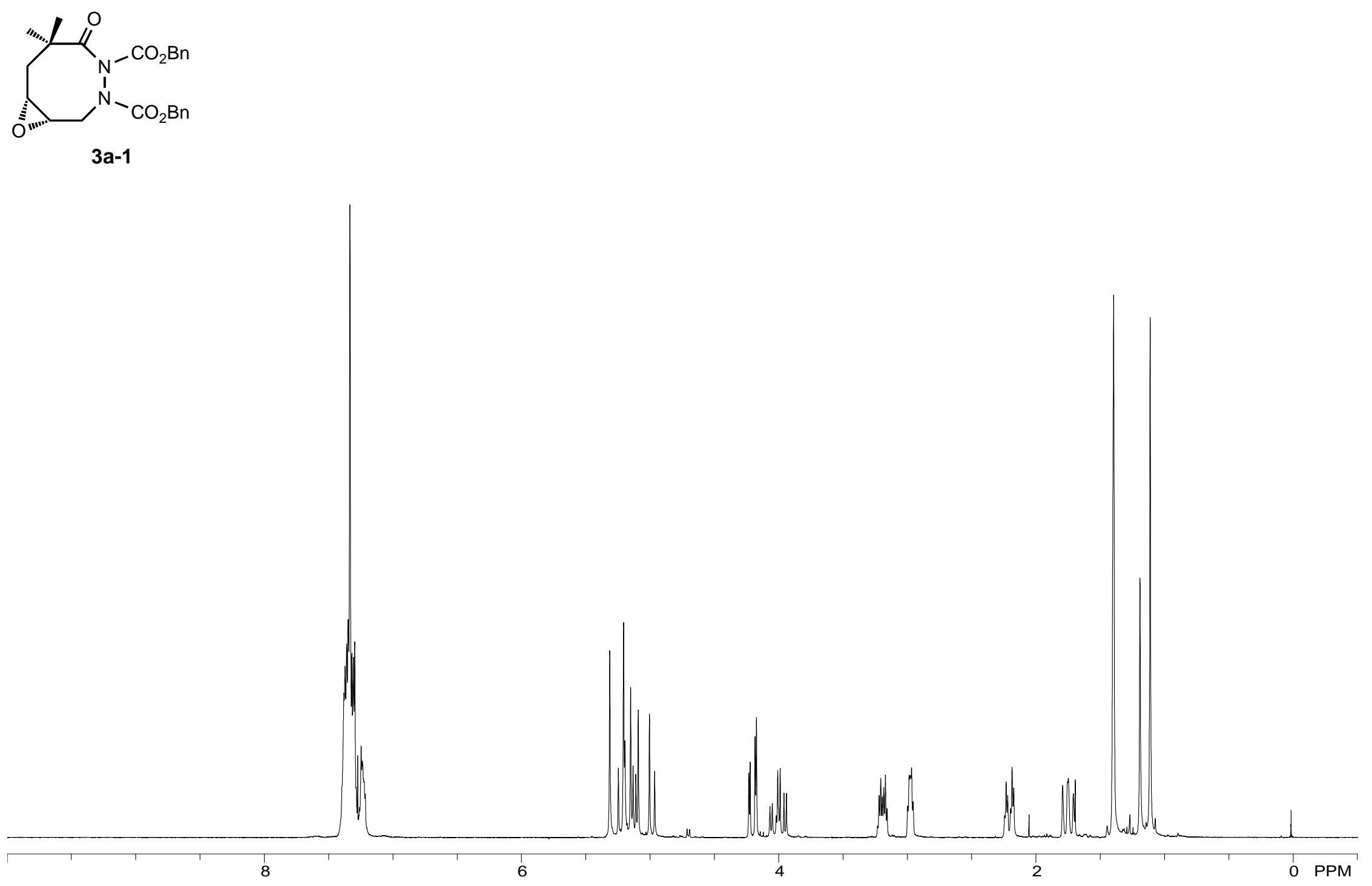

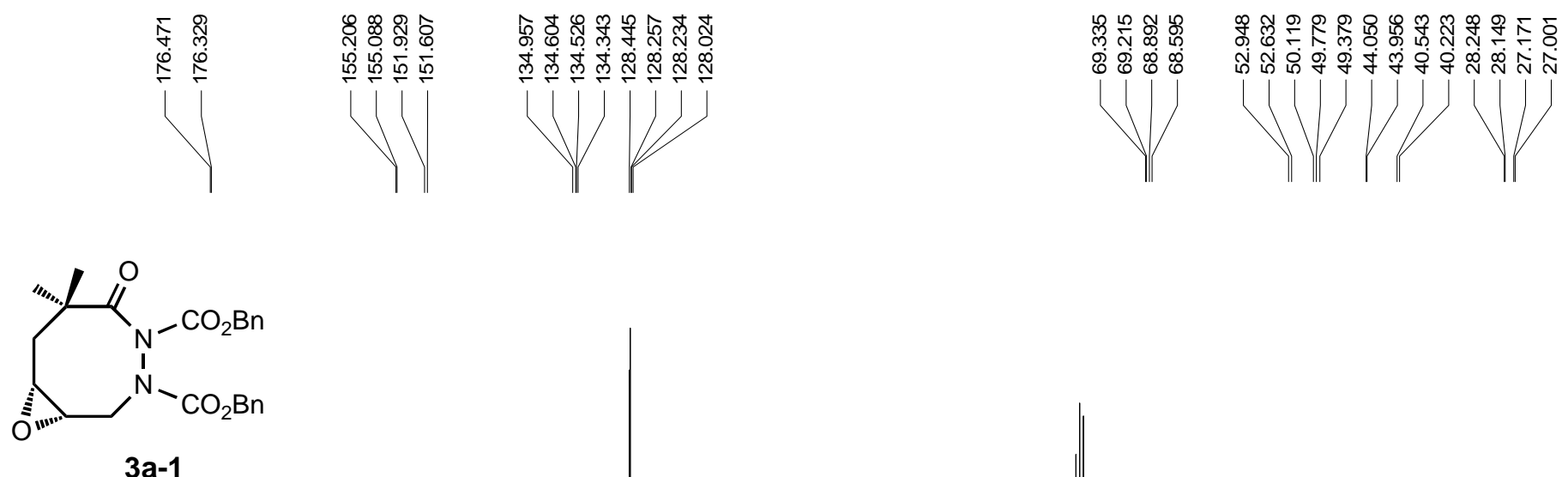

3a-1 
ť․

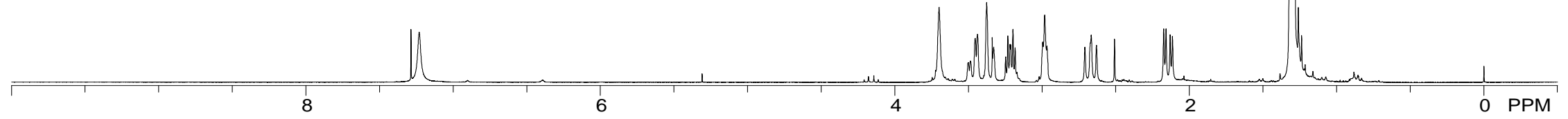




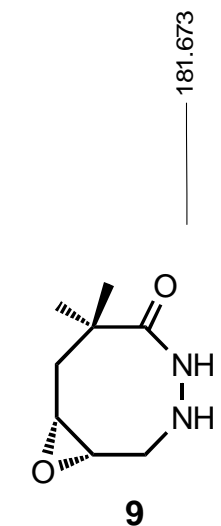

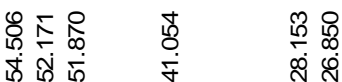




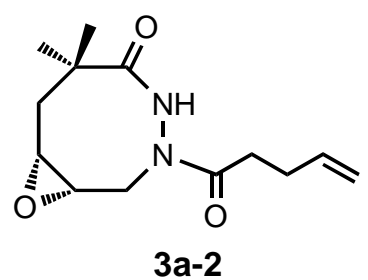


禺

兽

|

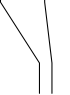

(N)

3a-2
용용

它心

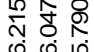

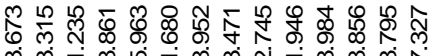

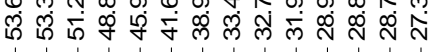
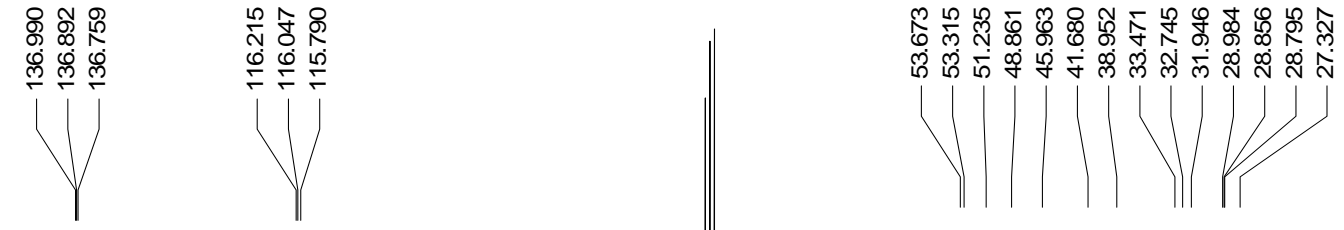

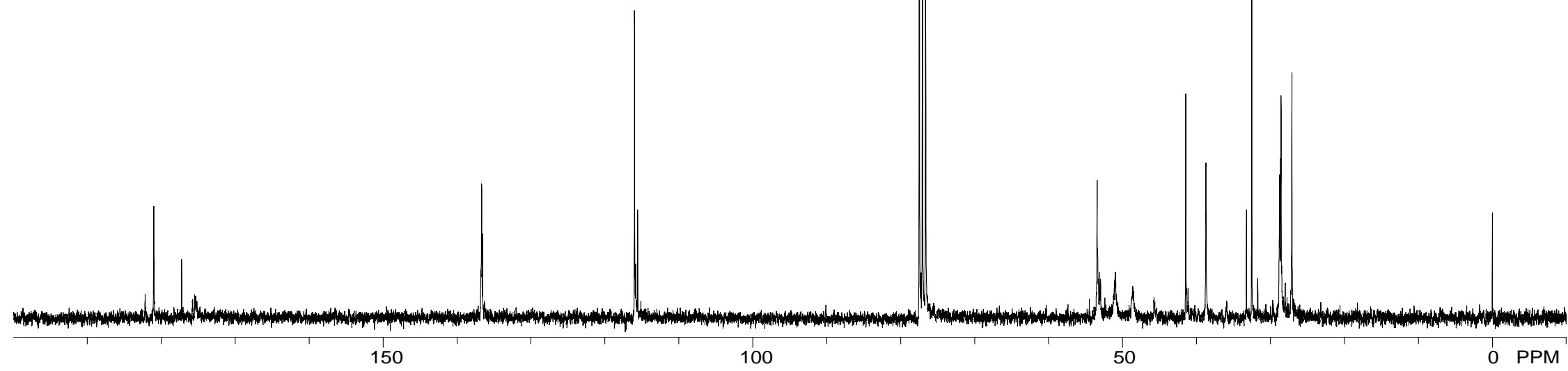


S37

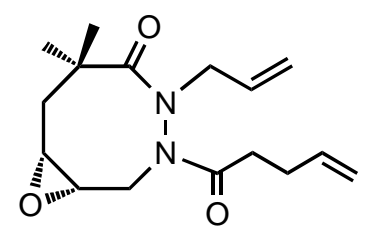

10

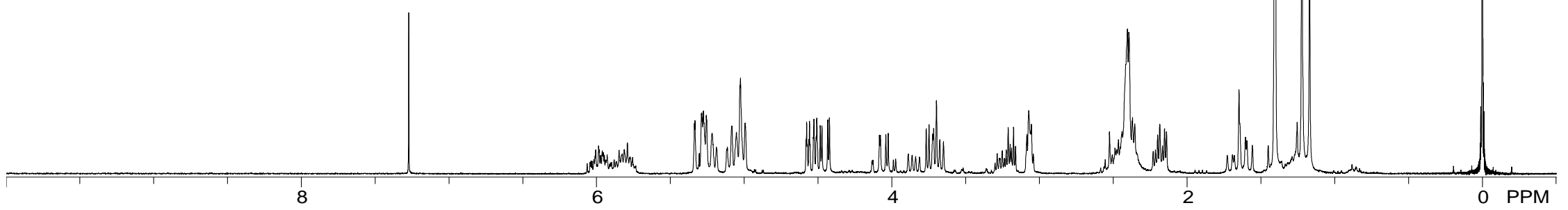




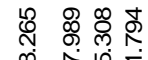

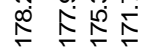

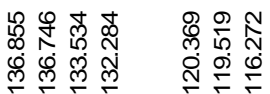

(3)

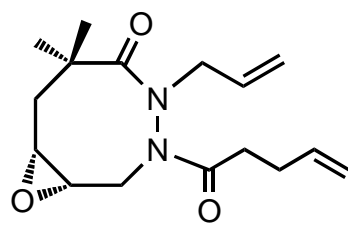

10

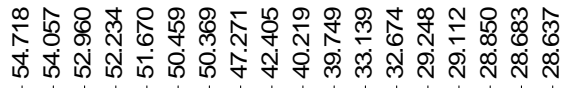



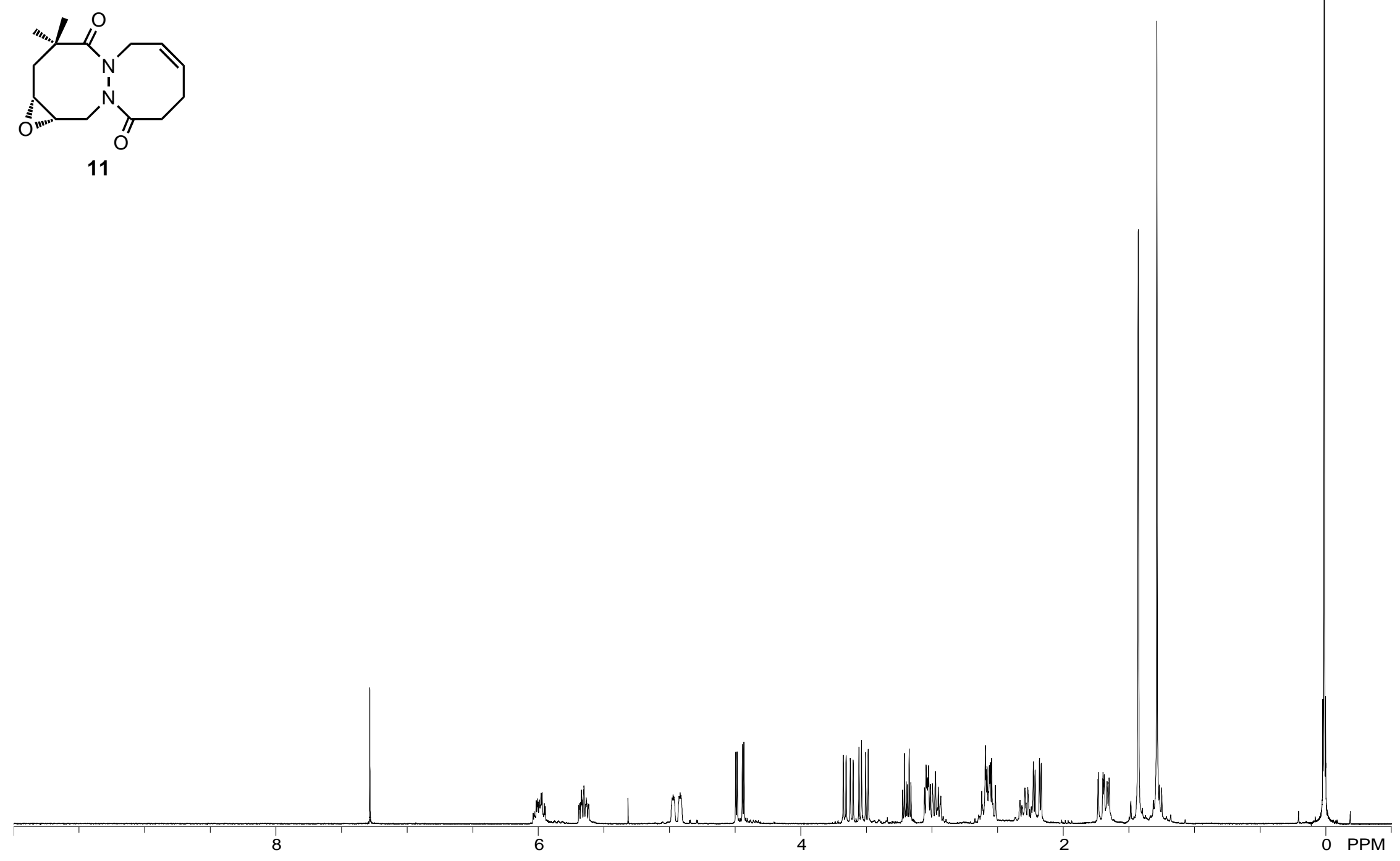

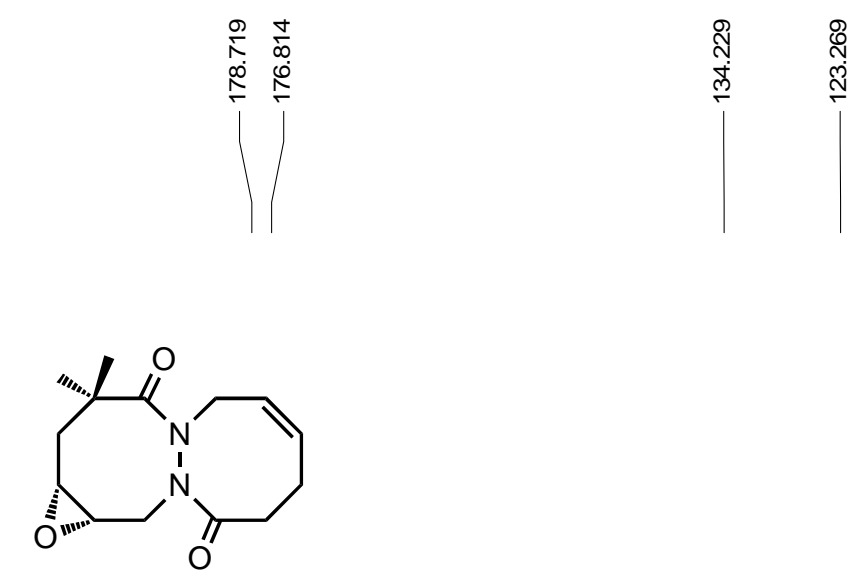

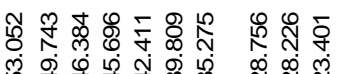

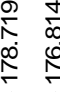

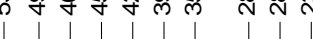

11

(1) 

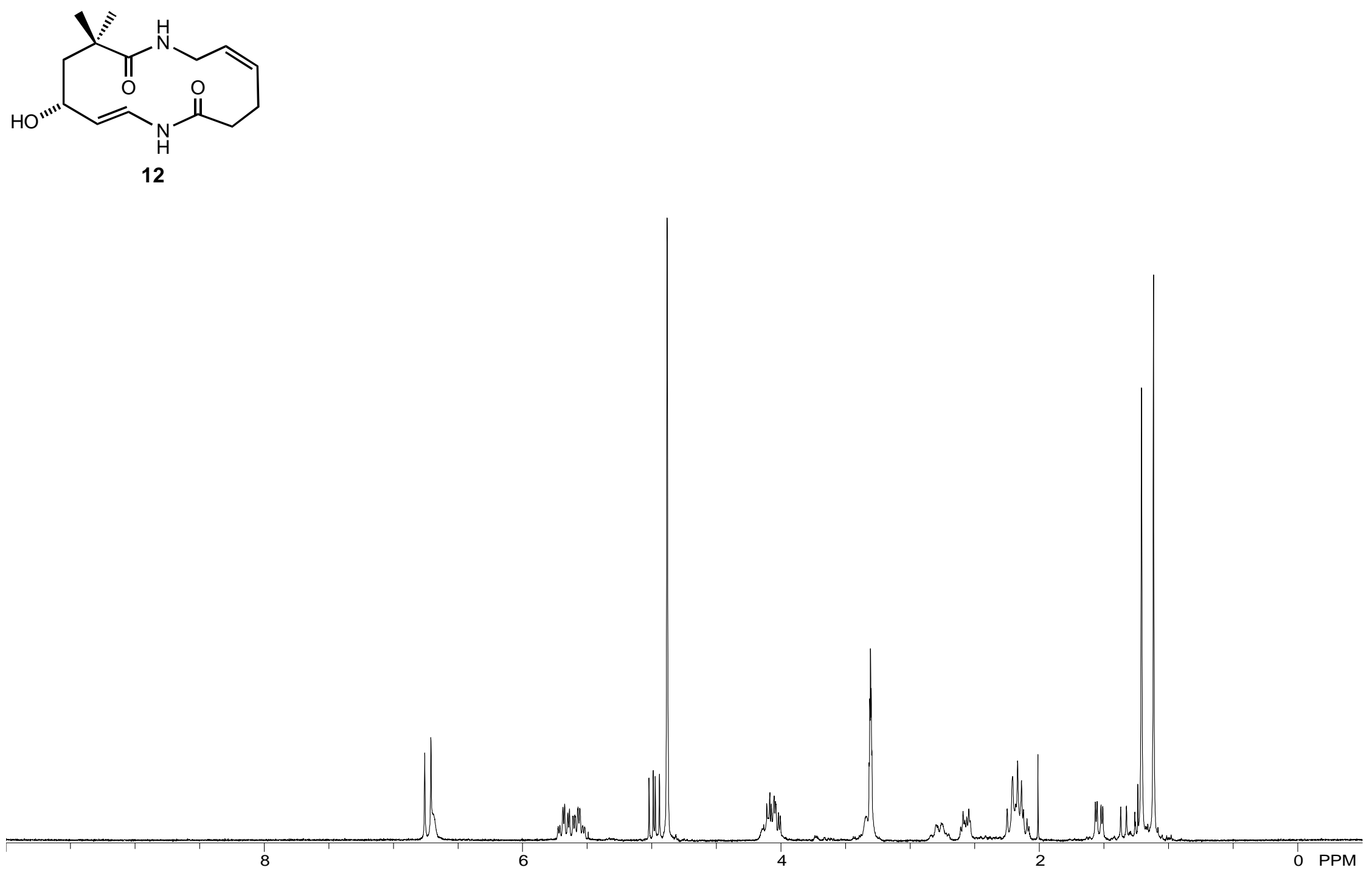

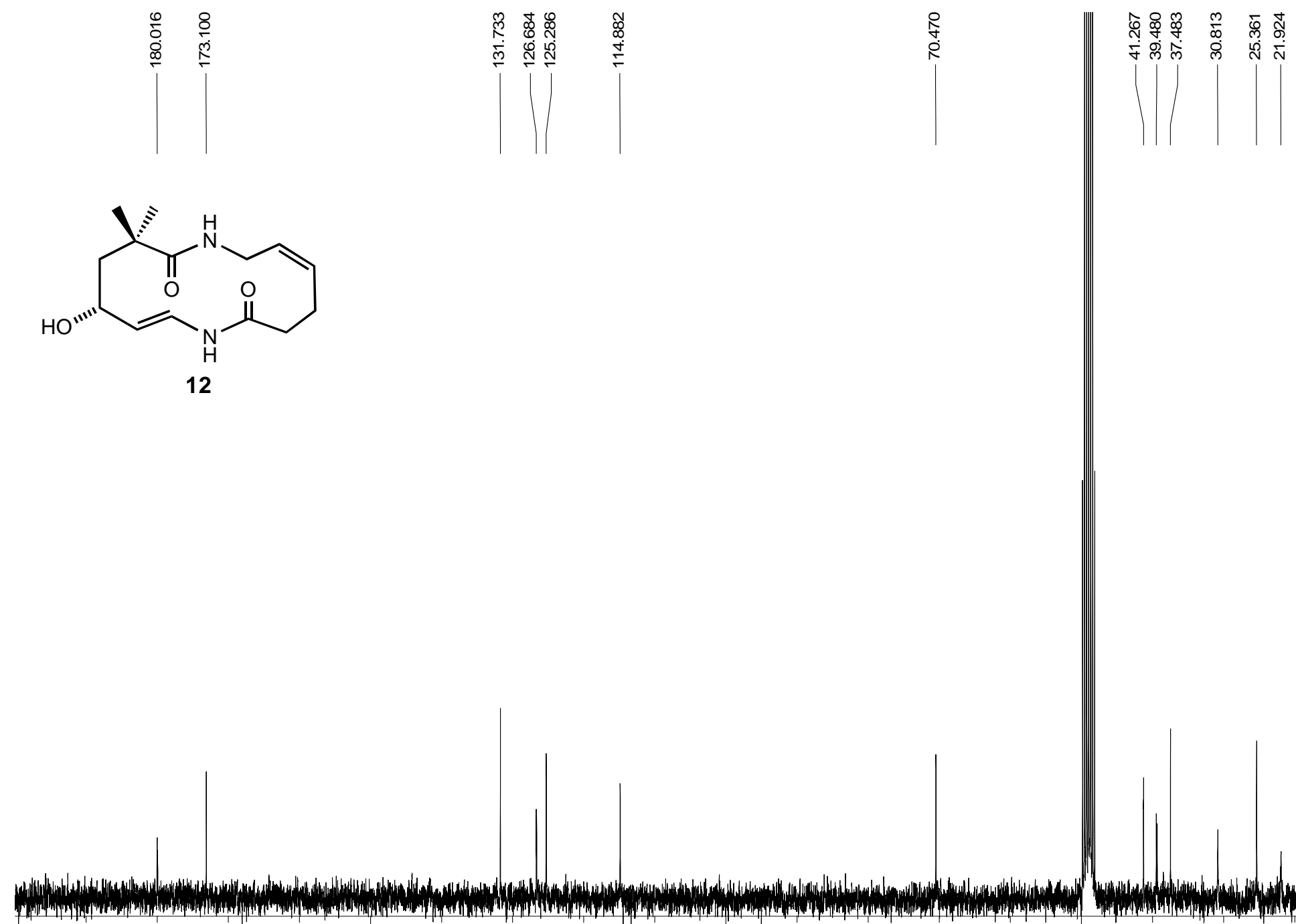


\section{X-Ray Crystal Structure Report for 12}

\section{Crystallographic Experimental Section}

\section{Data Collection}

A colorless crystal with approximate dimensions $0.36 \times 0.34 \times 0.18 \mathrm{~mm}^{3}$ was selected under oil under ambient conditions and attached to the tip of a nylon loop. The crystal was mounted in a stream of cold nitrogen at 100(2) $\mathrm{K}$ and centered in the X-ray beam by using a video camera.

The crystal evaluation and data collection were performed on a Bruker CCD-1000 diffractometer with $\mathrm{Mo} \mathrm{K}_{\alpha}(\lambda=0.71073 \AA)$ radiation and the diffractometer to crystal distance of $4.9 \mathrm{~cm}$.

The initial cell constants were obtained from three series of $\omega$ scans at different starting angles. Each series consisted of 20 frames collected at intervals of $0.3^{\circ}$ in a $6^{\circ}$ range about $\omega$ with the exposure time of 10 seconds per frame. A total of 51 reflections were obtained. The reflections were successfully indexed by an automated indexing routine built in the SMART program. The final cell constants were calculated from a set of 4169 strong reflections from the actual data collection.

The data were collected by using the hemisphere data collection routine. The reciprocal space was surveyed to the extent of a full sphere to a resolution of $0.80 \AA$. A total of 10809 data were harvested by collecting three sets of frames with $0.25^{\circ}$ scans in $\omega$ with an exposure time 90 sec per frame. These highly redundant datasets were corrected for Lorentz and polarization effects. The absorption correction was based on fitting a function to the empirical transmission surface as sampled by multiple equivalent measurements. [1]

\section{Structure Solution and Refinement}

The systematic absences in the diffraction data were uniquely consistent for the space group $P 2_{1} / n$ that yielded chemically reasonable and computationally stable results of refinement [1].

A successful solution by the direct methods provided most non-hydrogen atoms from the $E$-map. The remaining non-hydrogen atoms were located in an alternating series of least-squares cycles and difference Fourier maps. All non-hydrogen atoms were refined with anisotropic 
displacement coefficients. All hydrogen atoms were included in the structure factor calculation at idealized positions and were allowed to ride on the neighboring atoms with relative isotropic displacement coefficients.

The final least-squares refinement of 175 parameters against 2721 data resulted in residuals $R$ (based on $F^{2}$ for $I \geq 2 \sigma$ ) and $w R$ (based on $F^{2}$ for all data) of 0.0391 and 0.1067 , respectively. The final difference Fourier map was featureless.

The ORTEP diagram is drawn with $50 \%$ probability ellipsoids.

\section{References}

[1] Bruker-AXS. (2000-2003) SADABS V.2.05, SAINT V.6.22, SHELXTL V.6.10

\& SMART 5.622 Software Reference Manuals. Bruker-AXS, Madison, Wisconsin, USA. 


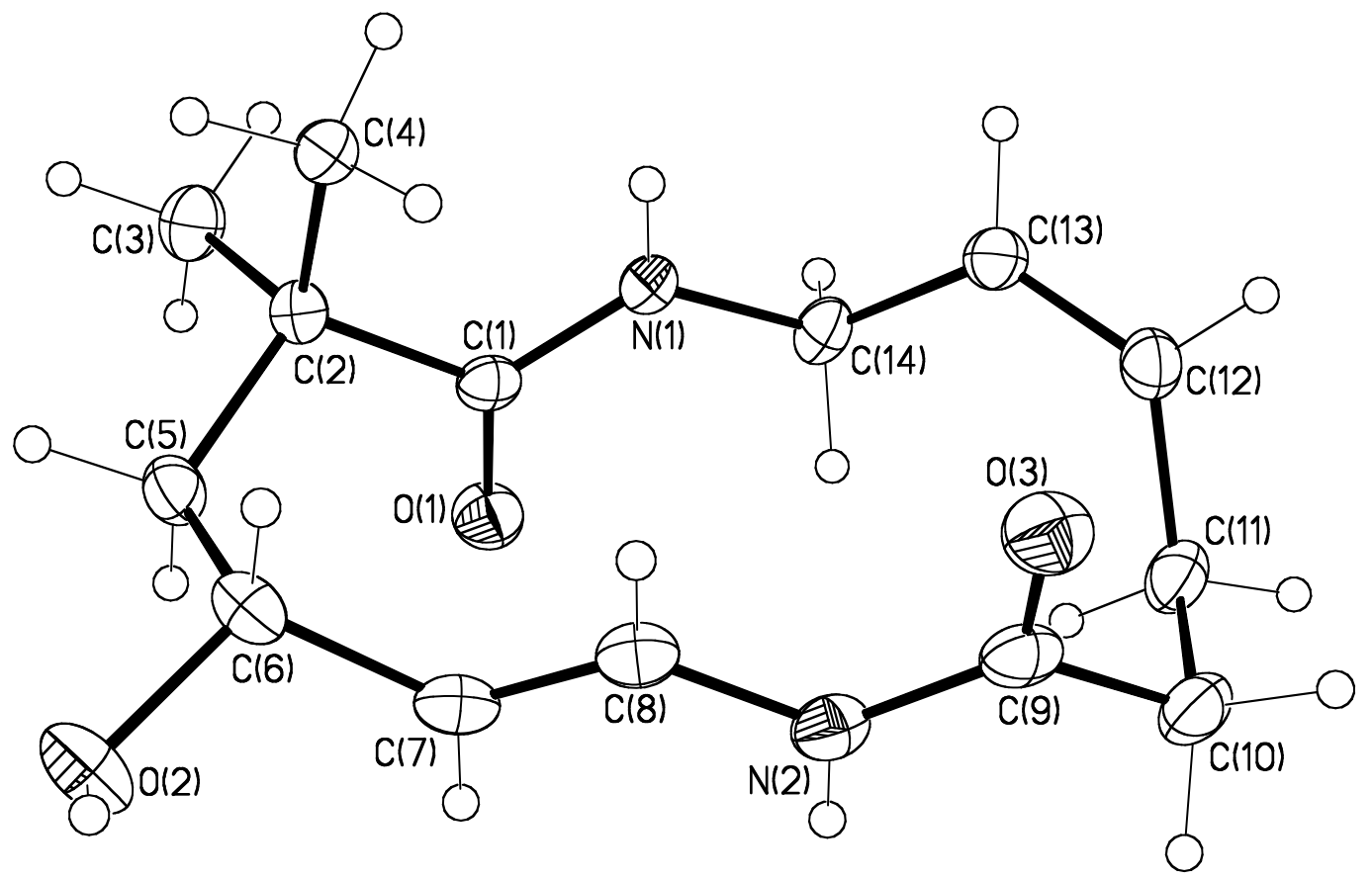

Figure 1. A thermal ellipsoid drawing of Lee017 (12). 


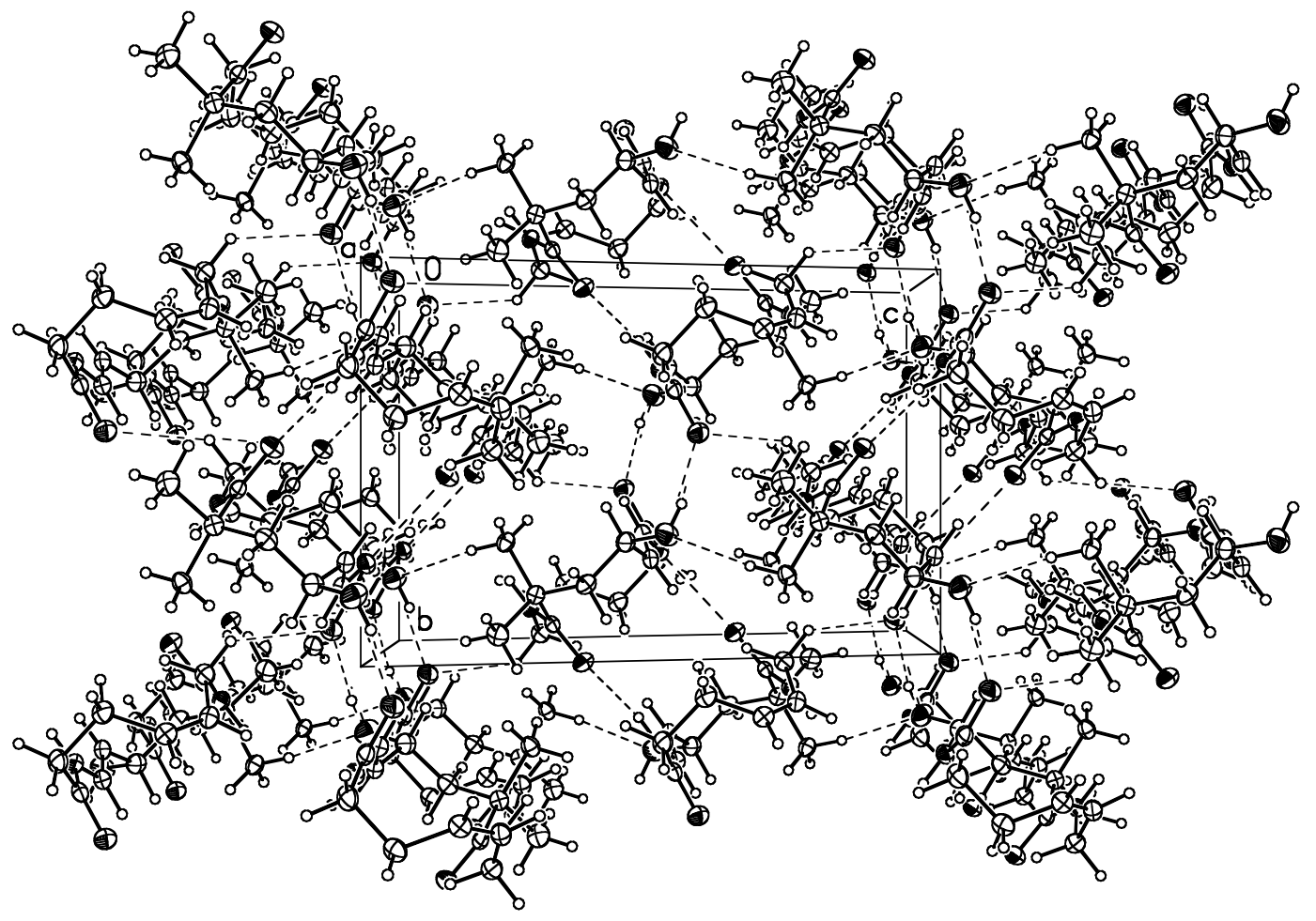

Figure 2. A packing diagram viewed along the a-axis. 


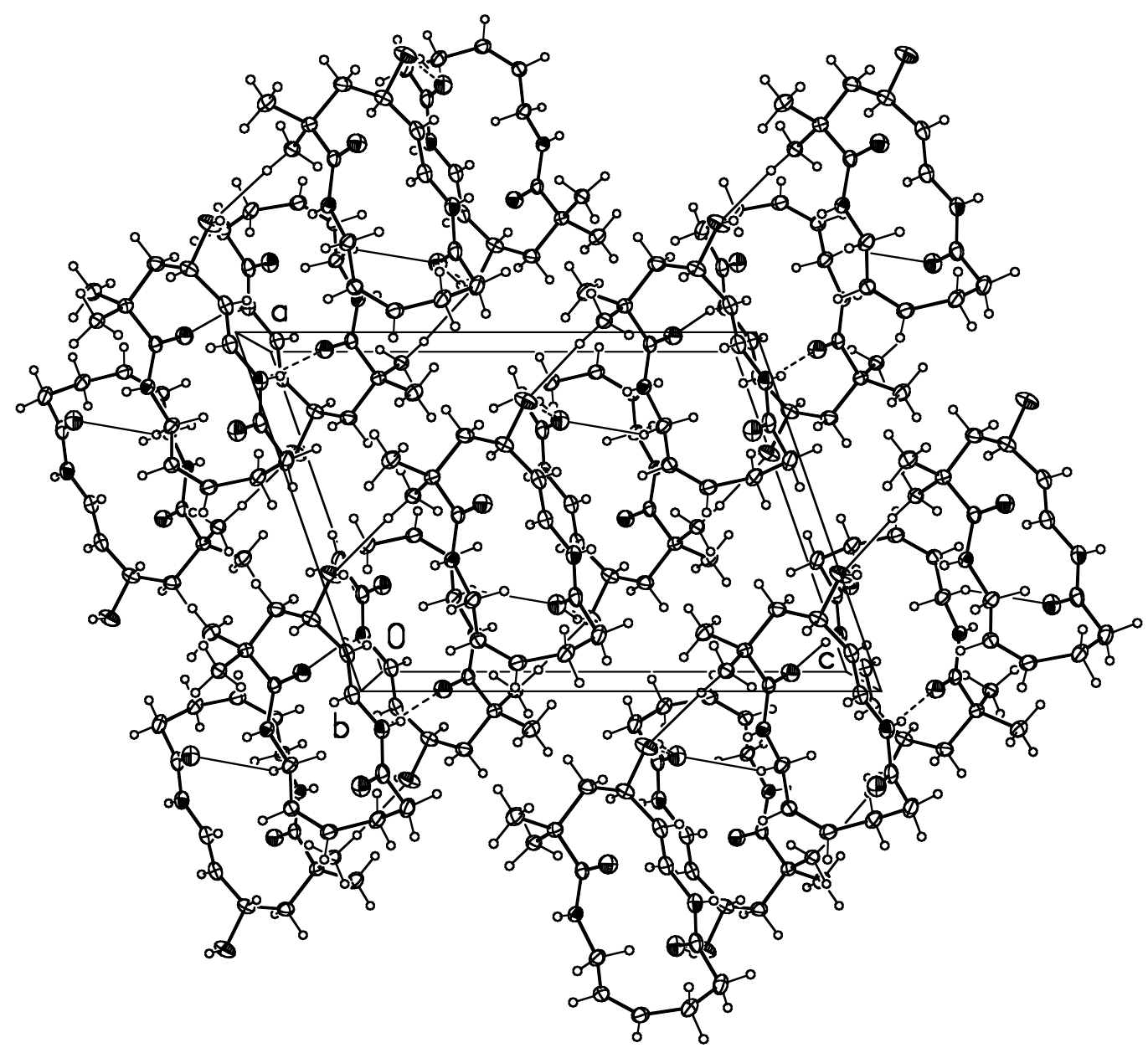

Figure 3. A packing diagram viewed along the b-axis. 


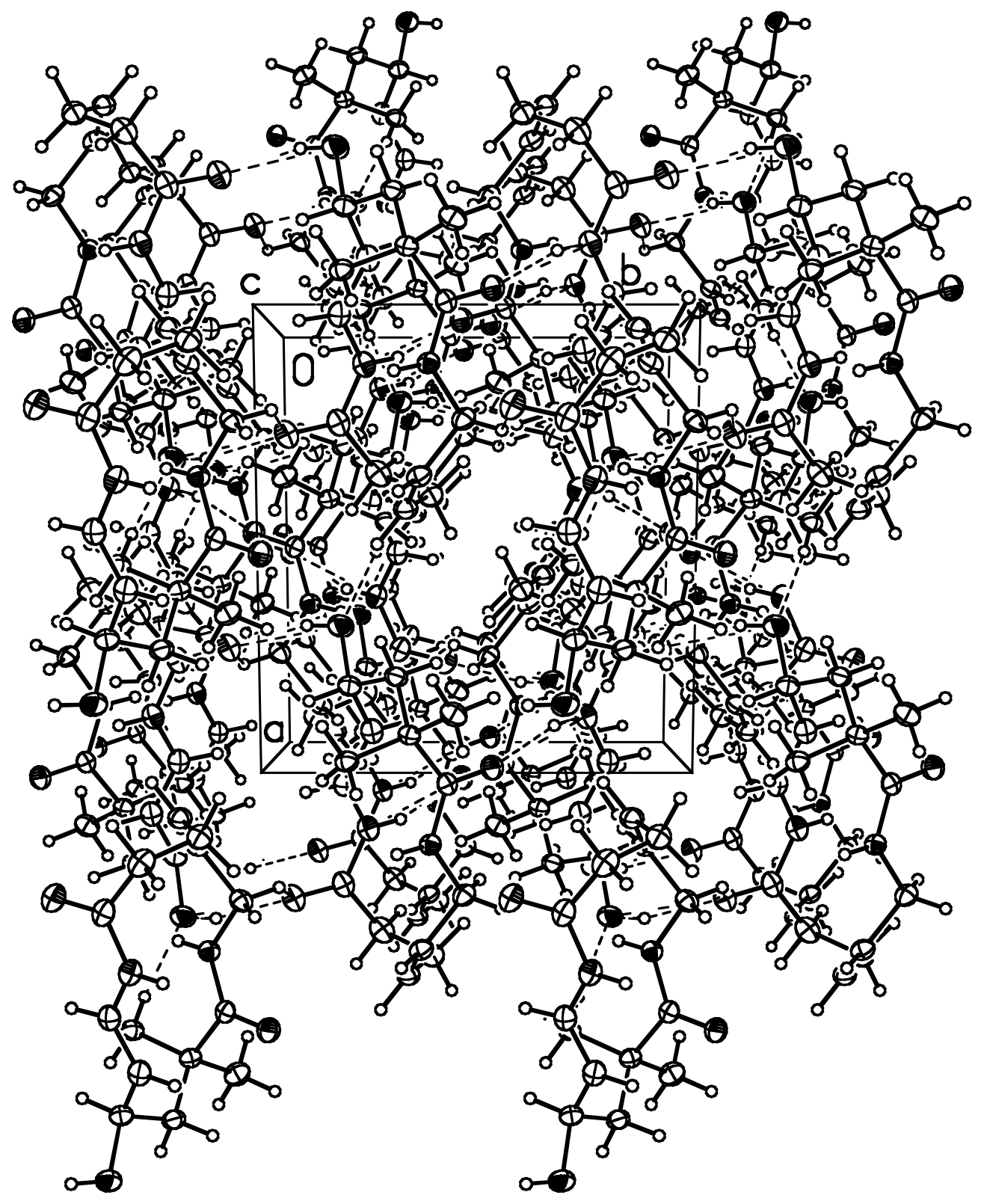

Figure 4. A packing diagram viewed along the c-axis. 
Table 1. Crystal data and structure refinement for lee 017.

Identification code

lee 017

Empirical formula

Formula weight

$\mathrm{C}_{14} \mathrm{H}_{22} \mathrm{~N}_{2} \mathrm{O}_{3}$

Temperature

266.34

Wavelength

$173(2) \mathrm{K}$

$0.71073 \AA$

Crystal system

Space group

Unit cell dimensions

Monoclinic

$\mathrm{P} 21 / \mathrm{n}$

$\mathrm{a}=10.6541(9) \AA$

$\alpha=90^{\circ}$.

$\mathrm{b}=9.4448(8) \AA$

$\mathrm{c}=14.5765(12) \AA$

$\beta=109.205(2)^{\circ}$.

Volume

1385.1(2) $\AA^{3}$

Z

4

Density (calculated)

$1.277 \mathrm{Mg} / \mathrm{m}^{3}$

Absorption coefficient

$0.090 \mathrm{~mm}^{-1}$

$\mathrm{F}(000)$

576

Crystal size

Theta range for data collection

Index ranges

Reflections collected

Independent reflections

Completeness to theta $=25.99^{\circ}$

Absorption correction

Max. and min. transmission

Refinement method

Data / restraints / parameters

Goodness-of-fit on $\mathrm{F}^{2}$

$0.36 \times 0.34 \times 0.18 \mathrm{~mm}^{3}$

2.08 to $25.99^{\circ}$.

$-12<=\mathrm{h}<=13,-11<=\mathrm{k}<=11,-17<=\mathrm{l}<=17$

10809

$2721[\mathrm{R}(\mathrm{int})=0.0258]$

$99.9 \%$

Multi-scan with SADABS

0.9840 and 0.9683

Full-matrix least-squares on $\mathrm{F}^{2}$

$2721 / 0 / 175$

1.008

Final R indices [I $>2 \operatorname{sigma}(\mathrm{I})]$

$\mathrm{R} 1=0.0391, \mathrm{wR} 2=0.1019$

$\mathrm{R}$ indices (all data)

$\mathrm{R} 1=0.0487, \mathrm{wR} 2=0.1067$

Largest diff. peak and hole

0.324 and -0.176 e. $\AA^{-3}$ 
Table 2. Atomic coordinates ( $\left.\times 10^{4}\right)$ and equivalent isotropic displacement parameters $\left(\AA^{2} \times 10^{3}\right)$ for lee017. $\mathrm{U}(\mathrm{eq})$ is defined as one third of the trace of the orthogonalized $\mathrm{U}^{\mathrm{ij}}$ tensor.

\begin{tabular}{lrrrr}
\hline & $\mathrm{x}$ & $\mathrm{y}$ & $\mathrm{z}$ & $\mathrm{U}(\mathrm{eq})$ \\
\hline $\mathrm{O}(1)$ & $-220(1)$ & $5349(1)$ & $1400(1)$ & $26(1)$ \\
$\mathrm{O}(2)$ & $-3211(1)$ & $1878(1)$ & $-122(1)$ & $33(1)$ \\
$\mathrm{O}(3)$ & $2721(1)$ & $755(1)$ & $686(1)$ & $29(1)$ \\
$\mathrm{N}(1)$ & $1354(1)$ & $3959(1)$ & $2399(1)$ & $19(1)$ \\
$\mathrm{N}(2)$ & $1240(1)$ & $2474(1)$ & $-3(1)$ & $23(1)$ \\
$\mathrm{C}(1)$ & $87(1)$ & $4337(1)$ & $1968(1)$ & $18(1)$ \\
$\mathrm{C}(2)$ & $-980(1)$ & $3498(2)$ & $2234(1)$ & $20(1)$ \\
$\mathrm{C}(3)$ & $-1458(2)$ & $4492(2)$ & $2889(1)$ & $28(1)$ \\
$\mathrm{C}(4)$ & $-456(1)$ & $2121(2)$ & $2785(1)$ & $21(1)$ \\
$\mathrm{C}(5)$ & $-2171(1)$ & $3186(2)$ & $1313(1)$ & $23(1)$ \\
$\mathrm{C}(6)$ & $-1939(1)$ & $2101(2)$ & $611(1)$ & $24(1)$ \\
$\mathrm{C}(7)$ & $-932(2)$ & $2587(2)$ & $171(1)$ & $24(1)$ \\
$\mathrm{C}(8)$ & $227(1)$ & $1943(2)$ & $317(1)$ & $23(1)$ \\
$\mathrm{C}(9)$ & $2466(2)$ & $1894(2)$ & $252(1)$ & $23(1)$ \\
$\mathrm{C}(10)$ & $3527(2)$ & $2777(2)$ & $51(1)$ & $27(1)$ \\
$\mathrm{C}(11)$ & $3992(2)$ & $3968(2)$ & $812(1)$ & $25(1)$ \\
$\mathrm{C}(12)$ & $4290(1)$ & $3435(2)$ & $1831(1)$ & $23(1)$ \\
$\mathrm{C}(13)$ & $3640(1)$ & $3747(2)$ & $2445(1)$ & $22(1)$ \\
$\mathrm{C}(14)$ & $2468(1)$ & $4718(2)$ & $2251(1)$ & $22(1)$ \\
& & & & \\
\hline
\end{tabular}


Table 3. Bond lengths $[\AA]$ and angles $\left[{ }^{\circ}\right]$ for lee 017 .

\begin{tabular}{lc}
\hline $\mathrm{O}(1)-\mathrm{C}(1)$ & $1.2357(16)$ \\
$\mathrm{O}(2)-\mathrm{C}(6)$ & $1.4385(16)$ \\
$\mathrm{O}(3)-\mathrm{C}(9)$ & $1.2316(18)$ \\
$\mathrm{N}(1)-\mathrm{C}(1)$ & $1.3362(17)$ \\
$\mathrm{N}(1)-\mathrm{C}(14)$ & $1.4629(17)$ \\
$\mathrm{N}(2)-\mathrm{C}(9)$ & $1.3508(19)$ \\
$\mathrm{N}(2)-\mathrm{C}(8)$ & $1.4019(19)$ \\
$\mathrm{C}(1)-\mathrm{C}(2)$ & $1.5366(18)$ \\
$\mathrm{C}(2)-\mathrm{C}(4)$ & $1.5335(19)$ \\
$\mathrm{C}(2)-\mathrm{C}(3)$ & $1.5393(19)$ \\
$\mathrm{C}(2)-\mathrm{C}(5)$ & $1.5426(18)$ \\
$\mathrm{C}(5)-\mathrm{C}(6)$ & $1.525(2)$ \\
$\mathrm{C}(6)-\mathrm{C}(7)$ & $1.491(2)$ \\
$\mathrm{C}(7)-\mathrm{C}(8)$ & $1.328(2)$ \\
$\mathrm{C}(9)-\mathrm{C}(10)$ & $1.510(2)$ \\
$\mathrm{C}(10)-\mathrm{C}(11)$ & $1.543(2)$ \\
$\mathrm{C}(11)-\mathrm{C}(12)$ & $1.500(2)$ \\
$\mathrm{C}(12)-\mathrm{C}(13)$ & $1.332(2)$ \\
$\mathrm{C}(13)-\mathrm{C}(14)$ & $1.500(2)$ \\
& \\
$\mathrm{C}(1)-\mathrm{N}(1)-\mathrm{C}(14)$ & $122.86(12)$ \\
$\mathrm{C}(9)-\mathrm{N}(2)-\mathrm{C}(8)$ & $122.99(12)$ \\
$\mathrm{O}(1)-\mathrm{C}(1)-\mathrm{N}(1)$ & $121.71(13)$ \\
$\mathrm{O}(1)-\mathrm{C}(1)-\mathrm{C}(2)$ & $120.86(12)$ \\
$\mathrm{N}(1)-\mathrm{C}(1)-\mathrm{C}(2)$ & $117.39(11)$ \\
$\mathrm{C}(4)-\mathrm{C}(2)-\mathrm{C}(1)$ & $113.41(11)$ \\
$\mathrm{C}(4)-\mathrm{C}(2)-\mathrm{C}(3)$ & $109.33(11)$ \\
$\mathrm{C}(1)-\mathrm{C}(2)-\mathrm{C}(3)$ & $105.37(11)$ \\
$\mathrm{C}(4)-\mathrm{C}(2)-\mathrm{C}(5)$ & $110.28(11)$ \\
$\mathrm{C}(1)-\mathrm{C}(2)-\mathrm{C}(5)$ & $110.07(11)$ \\
$\mathrm{C}(3)-\mathrm{C}(2)-\mathrm{C}(5)$ & $108.15(11)$ \\
$\mathrm{C}(6)-\mathrm{C}(5)-\mathrm{C}(2)$ & $116.42(11)$ \\
$\mathrm{O}(2)-\mathrm{C}(6)-\mathrm{C}(7)$ & $111.19(12)$ \\
$\mathrm{O}(2)-\mathrm{C}(6)-\mathrm{C}(5)$ & $105.99(11)$ \\
$\mathrm{C}(7)-\mathrm{C}(6)-\mathrm{C}(5)$ & $112.32(12)$ \\
$\mathrm{C}(8)-\mathrm{C}(7)-\mathrm{C}(6)$ & $123.49(13)$ \\
$\mathrm{C}(7)-\mathrm{C}(8)-\mathrm{N}(2)$ & $124.03(13)$ \\
$\mathrm{O}(3)-\mathrm{C}(9)-\mathrm{N}(2)$ & $121.70(14)$ \\
$\mathrm{O}(3)-\mathrm{C}(9)-\mathrm{C}(10)$ & $121.93(13)$ \\
$\mathrm{N}(2)-\mathrm{C}(9)-\mathrm{C}(10)$ & $116.17(13)$ \\
$\mathrm{C}(9)-\mathrm{C}(10)-\mathrm{C}(11)$ & $110.27(11)$ \\
$\mathrm{C}(12)-\mathrm{C}(11)-\mathrm{C}(10)$ & $1138(13)$ \\
$\mathrm{C}(13)-\mathrm{C}(12)-\mathrm{C}(11)$ & \\
$\mathrm{C}(12)-\mathrm{C}(13)-\mathrm{C}(14)$ & \\
$\mathrm{N}(1)-\mathrm{C}(14)-\mathrm{C}(13)$ & \\
& \\
\hline
\end{tabular}

Symmetry transformations used to generate equivalent atoms: 
Table 4. Anisotropic displacement parameters $\left(\AA^{2} \times 10^{3}\right)$ for lee017. The anisotropic displacement factor exponent takes the form: $-2 \pi^{2}\left[h^{2} a^{* 2} U^{11}+\ldots+2 h k a^{*} b^{*} U^{12}\right]$

\begin{tabular}{lcccccc}
\hline & $\mathrm{U}^{11}$ & $\mathrm{U}^{22}$ & $\mathrm{U}^{33}$ & $\mathrm{U}^{23}$ & $\mathrm{U}^{13}$ & $\mathrm{U}^{12}$ \\
\hline $\mathrm{O}(1)$ & $27(1)$ & $23(1)$ & $27(1)$ & $6(1)$ & $8(1)$ & $1(1)$ \\
$\mathrm{O}(2)$ & $24(1)$ & $31(1)$ & $33(1)$ & $-6(1)$ & $-4(1)$ & $3(1)$ \\
$\mathrm{O}(3)$ & $35(1)$ & $25(1)$ & $28(1)$ & $2(1)$ & $11(1)$ & $6(1)$ \\
$\mathrm{N}(1)$ & $19(1)$ & $21(1)$ & $19(1)$ & $2(1)$ & $8(1)$ & $-1(1)$ \\
$\mathrm{N}(2)$ & $29(1)$ & $23(1)$ & $19(1)$ & $2(1)$ & $9(1)$ & $3(1)$ \\
$\mathrm{C}(1)$ & $22(1)$ & $18(1)$ & $15(1)$ & $-3(1)$ & $7(1)$ & $1(1)$ \\
$\mathrm{C}(2)$ & $17(1)$ & $22(1)$ & $20(1)$ & $1(1)$ & $7(1)$ & $1(1)$ \\
$\mathrm{C}(3)$ & $25(1)$ & $31(1)$ & $30(1)$ & $-1(1)$ & $14(1)$ & $5(1)$ \\
$\mathrm{C}(4)$ & $19(1)$ & $24(1)$ & $20(1)$ & $4(1)$ & $6(1)$ & $-2(1)$ \\
$\mathrm{C}(5)$ & $17(1)$ & $25(1)$ & $25(1)$ & $4(1)$ & $5(1)$ & $2(1)$ \\
$\mathrm{C}(6)$ & $19(1)$ & $25(1)$ & $23(1)$ & $1(1)$ & $1(1)$ & $1(1)$ \\
$\mathrm{C}(7)$ & $30(1)$ & $23(1)$ & $15(1)$ & $-1(1)$ & $4(1)$ & $1(1)$ \\
$\mathrm{C}(8)$ & $28(1)$ & $22(1)$ & $18(1)$ & $-1(1)$ & $6(1)$ & $-1(1)$ \\
$\mathrm{C}(9)$ & $32(1)$ & $23(1)$ & $16(1)$ & $-3(1)$ & $9(1)$ & $4(1)$ \\
$\mathrm{C}(10)$ & $32(1)$ & $31(1)$ & $23(1)$ & $2(1)$ & $16(1)$ & $7(1)$ \\
$\mathrm{C}(11)$ & $26(1)$ & $27(1)$ & $27(1)$ & $3(1)$ & $15(1)$ & $1(1)$ \\
$\mathrm{C}(12)$ & $19(1)$ & $25(1)$ & $26(1)$ & $1(1)$ & $8(1)$ & $0(1)$ \\
$\mathrm{C}(13)$ & $20(1)$ & $26(1)$ & $20(1)$ & $2(1)$ & $6(1)$ & $-4(1)$ \\
$\mathrm{C}(14)$ & $22(1)$ & $24(1)$ & $24(1)$ & $-1(1)$ & $12(1)$ & $-4(1)$ \\
& & & & & & \\
\hline
\end{tabular}


Table 5. Hydrogen coordinates ( $\left.\times 10^{4}\right)$ and isotropic displacement parameters $\left(\AA^{2} \times 10^{3}\right)$ for lee 017.

\begin{tabular}{lrrrr}
\hline & $\mathrm{x}$ & $\mathrm{y}$ & $\mathrm{z}$ & $\mathrm{U}(\mathrm{eq})$ \\
\hline & & & & \\
$\mathrm{H}(2 \mathrm{O})$ & -3217 & 1085 & -385 & 49 \\
$\mathrm{H}(1)$ & 1525 & 3221 & 2790 & 23 \\
$\mathrm{H}(2 \mathrm{~N})$ & 1069 & 3218 & -387 & 28 \\
$\mathrm{H}(3 \mathrm{~A})$ & -718 & 4696 & 3483 & 42 \\
$\mathrm{H}(3 \mathrm{~B})$ & -2178 & 4035 & 3058 & 42 \\
$\mathrm{H}(3 \mathrm{C})$ & -1783 & 5378 & 2541 & 42 \\
$\mathrm{H}(4 \mathrm{~A})$ & -22 & 1552 & 2414 & 32 \\
$\mathrm{H}(4 \mathrm{~B})$ & -1199 & 1586 & 2869 & 32 \\
$\mathrm{H}(4 \mathrm{C})$ & 186 & 2346 & 3423 & 32 \\
$\mathrm{H}(5 \mathrm{~A})$ & -2919 & 2851 & 1517 & 27 \\
$\mathrm{H}(5 \mathrm{~B})$ & -2450 & 4086 & 956 & 27 \\
$\mathrm{H}(6)$ & -1631 & 1192 & 966 & 29 \\
$\mathrm{H}(7)$ & -1129 & 3400 & -235 & 28 \\
$\mathrm{H}(8)$ & 377 & 1070 & 660 & 27 \\
$\mathrm{H}(10 \mathrm{~A})$ & 3171 & 3195 & -607 & 33 \\
$\mathrm{H}(10 \mathrm{~B})$ & 4293 & 2168 & 71 & 33 \\
$\mathrm{H}(11 \mathrm{~A})$ & 4799 & 4416 & 749 & 30 \\
$\mathrm{H}(11 \mathrm{~B})$ & 3290 & 4701 & 680 & 30 \\
$\mathrm{H}(12)$ & 5022 & 2807 & 2064 & 27 \\
$\mathrm{H}(13)$ & 3949 & 3311 & 3066 & 26 \\
$\mathrm{H}(14 \mathrm{~A})$ & 2708 & 5542 & 2694 & 26 \\
$\mathrm{H}(14 \mathrm{~B})$ & 2207 & 5072 & 1574 & 26 \\
& & & & \\
\hline
\end{tabular}


Table 6. Torsion angles $\left[{ }^{\circ}\right]$ for lee 017.

\begin{tabular}{lc}
\hline $\mathrm{C}(14)-\mathrm{N}(1)-\mathrm{C}(1)-\mathrm{O}(1)$ & $1.1(2)$ \\
$\mathrm{C}(14)-\mathrm{N}(1)-\mathrm{C}(1)-\mathrm{C}(2)$ & $-176.56(11)$ \\
$\mathrm{O}(1)-\mathrm{C}(1)-\mathrm{C}(2)-\mathrm{C}(4)$ & $168.89(12)$ \\
$\mathrm{N}(1)-\mathrm{C}(1)-\mathrm{C}(2)-\mathrm{C}(4)$ & $-13.38(17)$ \\
$\mathrm{O}(1)-\mathrm{C}(1)-\mathrm{C}(2)-\mathrm{C}(3)$ & $-71.57(15)$ \\
$\mathrm{N}(1)-\mathrm{C}(1)-\mathrm{C}(2)-\mathrm{C}(3)$ & $106.16(13)$ \\
$\mathrm{O}(1)-\mathrm{C}(1)-\mathrm{C}(2)-\mathrm{C}(5)$ & $44.82(16)$ \\
$\mathrm{N}(1)-\mathrm{C}(1)-\mathrm{C}(2)-\mathrm{C}(5)$ & $-137.45(12)$ \\
$\mathrm{C}(4)-\mathrm{C}(2)-\mathrm{C}(5)-\mathrm{C}(6)$ & $-54.79(15)$ \\
$\mathrm{C}(1)-\mathrm{C}(2)-\mathrm{C}(5)-\mathrm{C}(6)$ & $71.08(15)$ \\
$\mathrm{C}(3)-\mathrm{C}(2)-\mathrm{C}(5)-\mathrm{C}(6)$ & $-174.29(12)$ \\
$\mathrm{C}(2)-\mathrm{C}(5)-\mathrm{C}(6)-\mathrm{O}(2)$ & $173.79(11)$ \\
$\mathrm{C}(2)-\mathrm{C}(5)-\mathrm{C}(6)-\mathrm{C}(7)$ & $-64.60(16)$ \\
$\mathrm{O}(2)-\mathrm{C}(6)-\mathrm{C}(7)-\mathrm{C}(8)$ & $-125.47(14)$ \\
$\mathrm{C}(5)-\mathrm{C}(6)-\mathrm{C}(7)-\mathrm{C}(8)$ & $115.94(15)$ \\
$\mathrm{C}(6)-\mathrm{C}(7)-\mathrm{C}(8)-\mathrm{N}(2)$ & $-172.76(13)$ \\
$\mathrm{C}(9)-\mathrm{N}(2)-\mathrm{C}(8)-\mathrm{C}(7)$ & $171.82(13)$ \\
$\mathrm{C}(8)-\mathrm{N}(2)-\mathrm{C}(9)-\mathrm{O}(3)$ & $9.1(2)$ \\
$\mathrm{C}(8)-\mathrm{N}(2)-\mathrm{C}(9)-\mathrm{C}(10)$ & $-165.88(12)$ \\
$\mathrm{O}(3)-\mathrm{C}(9)-\mathrm{C}(10)-\mathrm{C}(11)$ & $-98.63(16)$ \\
$\mathrm{N}(2)-\mathrm{C}(9)-\mathrm{C}(10)-\mathrm{C}(11)$ & $76.38(16)$ \\
$\mathrm{C}(9)-\mathrm{C}(10)-\mathrm{C}(11)-\mathrm{C}(12)$ & $47.89(16)$ \\
$\mathrm{C}(10)-\mathrm{C}(11)-\mathrm{C}(12)-\mathrm{C}(13)$ & $-114.00(17)$ \\
$\mathrm{C}(11)-\mathrm{C}(12)-\mathrm{C}(13)-\mathrm{C}(14)$ & $-0.7(2)$ \\
$\mathrm{C}(1)-\mathrm{N}(1)-\mathrm{C}(14)-\mathrm{C}(13)$ & $-155.28(12)$ \\
$\mathrm{C}(12)-\mathrm{C}(13)-\mathrm{C}(14)-\mathrm{N}(1)$ & $123.40(15)$ \\
& \\
&
\end{tabular}

Symmetry transformations used to generate equivalent atoms: 
Table 7. Hydrogen bonds for lee017 [ $\left[\AA\right.$ and $\left.^{\circ}\right]$.

\begin{tabular}{lcccc}
\hline $\mathrm{D}-\mathrm{H} \ldots \mathrm{A}$ & $\mathrm{d}(\mathrm{D}-\mathrm{H})$ & $\mathrm{d}(\mathrm{H} \ldots \mathrm{A})$ & $\mathrm{d}(\mathrm{D} \ldots \mathrm{A})$ & $<(\mathrm{DHA})$ \\
\hline $\mathrm{O}(2)-\mathrm{H}(2 \mathrm{O}) \ldots \mathrm{O}(3) \# 1$ & 0.84 & 1.91 & $2.7230(16)$ & 162.7 \\
$\mathrm{~N}(2)-\mathrm{H}(2 \mathrm{~N}) \ldots \mathrm{O}(1) \# 2$ & 0.88 & 1.99 & $2.8467(16)$ & 164.8
\end{tabular}

Symmetry transformations used to generate equivalent atoms:

$\# 1-\mathrm{x},-\mathrm{y},-\mathrm{z} \quad \# 2-\mathrm{x},-\mathrm{y}+1,-\mathrm{z}$ 